\title{
Designing Green Communication Systems for Smart and Connected Communities via Dynamic Spectrum Access
}

\author{
VIJAY K. SHAH, University of Kentucky, USA \\ SHAMEEK BHATTACHARJEE, Western Michigan University, USA \\ SIMONE SILVESTRI, University of Kentucky, USA \\ SAJAL K. DAS, Missouri University of Science and Technology, USA
}

\begin{abstract}
Smart and connected communities (SCCs) are emerging as a novel paradigm that allows the community residents to be connected with surrounding environments through smart technologies. However, there remain important challenges to fully exploit the potential of SCCs in improving societal well-being and prosperity. In particular, there is a need for designing green communication systems that are also capable of providing high quality of service (QoS) to distribute and collect information to and from SCCs. However, simultaneously satisfying both of these criteria is difficult due to varying demands posed by heterogeneous sensing modalities, lack of dedicated infrastructure in rural/sub-urban areas, and certain sustainability constraints. While low-power short-range technologies often fail to achieve high QoS, using 3G or 4G technologies (LTE, LTEA, GSM) for SCCs will eventually face spectrum scarcity and cross technology interference. In recent times, Dynamic spectrum access (DSA) has been proposed as a solution to overcome policy constraints and improve spectrum scarcity by spectrum sharing. In this article, we show that harnessing DSA in the context of SCCs can also achieve notable benefits in terms of energy efficiency and sustainability. Specifically, we propose a novel architecture for designing sustainable SCCs using a small-scale DSA-enabled overlay network that improves end-to-end energy efficiency of the network while guaranteeing QoS. We also propose a dynamic spectrum band selection approach that intelligently matches any message requirement to a suitable band type by exploiting distinct electro-magnetic characteristics of various bands. Since data generated in SCCs are typically valuable only when delivered within a certain hard (or soft) deadline, we formulate a linear optimization problem for determining the most energy-efficient path that ensures a delivery time within the hard deadline. After proving that such a problem is NP-Hard, we propose an exact pseudo-polynomial time dynamic programming algorithm to solve it followed by a polynomial time greedy heuristic. Additionally, we formulate a non-linear optimization problem to find the optimal path when the message delivery time is defined as a soft deadline and extend our greedy heuristic to handle soft deadlines. Compared to the homogeneous band access approaches that opportunistically access free channels within a given spectrum band, our extensive simulation study shows that the proposed dynamic multi-band selection approach significantly improves the achievable energy efficiency while meeting various hard and soft deadlines.
\end{abstract}

CCS Concepts: • Networks $\rightarrow$ Cognitive radios; • Hardware $\rightarrow$ Energy distribution; Impact on the environment; Sensor applications and deployments; Emerging technologies; • Computer systems organization $\rightarrow$ Embedded and cyber-physical systems;

This research is partially supported by the NSF grants CNS-1545037, CNS-1545050, and NeTS-1818942 and NATO Science for Peace and Security grant G4936.

Authors' addresses: V. K. Shah and S. Silvestri, Computer Science Department, University of Kentucky, Lexington, USA; emails: vijay.shah@uky.edu, silvestri@cs.uky.edu; S. Bhattacharjee, Computer Science Department, Western Michigan University, Kalamazoo, USA; email: shameek.bhattacharjee@wmich.edu; S. K. Das, Computer Science Department, Missouri University of Science and Technology, Rolla, USA; email: sdas@mst.edu.

Permission to make digital or hard copies of all or part of this work for personal or classroom use is granted without fee provided that copies are not made or distributed for profit or commercial advantage and that copies bear this notice and the full citation on the first page. Copyrights for components of this work owned by others than ACM must be honored. Abstracting with credit is permitted. To copy otherwise, or republish, to post on servers or to redistribute to lists, requires prior specific permission and/or a fee. Request permissions from permissions@acm.org.

(C) 2018 Association for Computing Machinery.

1550-4859/2018/11-ART31 \$15.00

https://doi.org/10.1145/3274284 
Additional Key Words and Phrases: Green communication, dynamic spectrum access, smart and connected communities, energy efficiency, internet of things, band selection

\section{ACM Reference format:}

Vijay K. Shah, Shameek Bhattacharjee, Simone Silvestri, and Sajal K. Das. 2018. Designing Green Communication Systems for Smart and Connected Communities via Dynamic Spectrum Access. ACM Trans. Sen. Netw. 14, 3-4, Article 31 (November 2018), 32 pages.

https://doi.org/10.1145/3274284

\section{INTRODUCTION}

Smart and connected communities (SCCs) is a novel paradigm that is increasingly attracting attention from government agencies (e.g., the National Science Foundation) as well as the research community [44]. The emergence of SCCs is a result of tremendous technological transformation that is allowing the community residents to have access to intelligent services provided with the data gathered through smart and pervasive sensing technologies. While examples of urban SCCs include smart homes [45] equipped with temperature sensors, light sensors, air quality sensors, and smart meters [33], examples of rural SCCs include smart agricultural fields equipped with moisture sensors, soil monitors, and wind sensors [50]. In this article, we define a sensor block as a generic unit generating data, and a certain SCC may contain hundreds (rural) and thousands (urban) of such sensor blocks.

To realize SCCs, it is essential to provide ubiquitous and efficient connectivity of sensor blocks with the data fusion centers that are responsible for data gathering, analytics, and dissemination for efficient decision making and ultimately improving societal well-being and prosperity through targeted intelligent services [63]. Therefore, a communication infrastructure is required to provide connectivity and guaranteed delivery of the data generated from various sensor blocks to their respective decision-making units (e.g., to a data fusion center). Such generated data often have quality of service (QoS) requirements, depending on the specific application. In this article, QoS is defined in terms of a pre-specified hard or soft deadline. A hard deadline defines the maximum time during which the information is deemed valuable, and thus it needs to be delivered to the decisionmaking unit within that deadline. Conversely, under a soft deadline requirement the benefit of receiving a message decreases over time according to a penalty function (see Section 6 for details).

In addition to providing the desired QoS requirements, there is a need for the communication infrastructure for SCCs to be energy efficient. Indeed, energy efficiency is an important concern for building sustainable SCCs, since Information and Communication Technologies (ICT) contribute up to $10 \%$ of the world's overall energy consumption, and this amount is expected to double every five years [43]. According to a recent report [65], 830M tons of $\mathrm{CO}_{2}$ are produced to generate the energy required by ICT devices yearly, and this amount is expected to double by 2020 . Furthermore, ICT industries spend tens of millions of dollars in the annual operational costs, such as electricity bills [49]. Hence, designing energy-efficient communication systems will not only have great ecological benefits and social responsibility in fighting climate change but also have significant economic benefits. This is why there is mounting pressure to design "Green ICT communication systems," where energy consumption is considered as first-class network system property [43]. Several international research projects such EARTH [20], Green Radio [19], OPERANet [4], and eWIN [43] are dedicated to energy-efficient wireless communications.

\subsection{Technical Challenges and Opportunities}

The realization of a communication infrastructure that is green and also provides high QoS for sustainable SCCs faces economic and sustainability hurdles for both rural (non-urban) and urban 
areas around the world. First, it is not cost-effective for ICT providers to invest in dedicated communication infrastructures, such as base stations, access points with backhaul links, and spectrum licenses in non-urban areas, due to low revenue returns compared to the high initial investment [5, 21]. Hence, sensor blocks planned in non-urban areas are usually far away from the data fusion center or the nearest backhaul link. Second, while certain real-time delay critical decisions having a local area scope may use fog computing [8], which may also require a dedicated infrastructure, many decisions need data gathered over a wider area over a longer-term horizon. Therefore, even in urban areas, the collected data need to travel larger distances between the sensor blocks and data fusion centers. Finally, even if the existence of a communication infrastructure is assumed (as in urban areas), distributing data from thousands of sensor blocks using the legacy infrastructure is likely to burden the existing wired networks and, in turn, requires deployment of more routers or switches, which would require additional electric power/wiring and, hence, exacerbates the problem of energy sustainability.

To address the above challenges related to the infrastructure costs (rural/sub-urban areas) and burdening the wired networks (urban areas), the approach adopted in this article proposes the use of a network of fixed (or mobile) cyber-physical public infrastructure mounted with wireless devices (or radios) [47, 54, 63], such as the road side assistance units (RSUs) [58], private smart vehicles, and public transportation. These are connected through wireless links and do not require additional dedicated communication infrastructures [10, 66]. Such an architecture implying delay tolerant communications [46] is acceptable for various decision and analytics applications in SCCs that do not require short real-time delivery deadlines. For example, smart meter data in a power grid usually have 0.5 - or 1-hour delivery deadlines for demand forecast [61], whereas basic agricultural sensor data such as wind or moisture sensors may tolerate higher time delays up to several hours.

However, relying on wireless connectivity for realizing communication infrastructure makes the wireless spectrum a critical resource for the success of SCCs. The current wireless communication technologies for SCCs typically include low-power short-range technologies (e.g., ZigBee, Bluetooth, WiFi, 6LowPAN using 2.4GHz), and 3/4GPP standards (e.g., GSM, LTE/LTE-A using $900,1700-2100 \mathrm{MHz}$ ). Low-power technologies, although seemingly energy efficient, have a limited transmission coverage [18]. Since the data often need to travel larger distances in the context of SCCs as discussed earlier, a large number of devices need to be deployed to form a multi-hop network [33], such that a high QoS is guaranteed. However, this kind of communication network exacerbates the problem of both energy efficiency and monetary cost. Conversely, while 3/4GPP technologies (dominant in urban areas) provide higher transmission coverage, they come at a cost of higher energy expenditure [29] due to two major reasons. First, the co-existence of increasingly myriad devices in the same spectrum will cause cross technology interference and thereby decreased Signal-to-Noise Ratio (SINR). This necessitates either increased transmit power or higher number of re-transmissions, thus worsening the energy expenditure. Second, the static nature of legacy spectrum access policy does not provide the flexibility to handle data traffic with heterogeneous demands (e.g., message sizes and message delivery deadlines) that may be generated from several sensor blocks. Such homogeneity of the spectrum access policy triggers the disadvantage of resource over-provisioning or under-provisioning that, again, negatively impacts the achievable energy efficiency (refer to Section 4 for details).

In the past decade, Dynamic Spectrum Access (DSA) $[2,59]$ has emerged as an enabling paradigm that allows certain DSA equipped wireless devices, also termed secondary users, to opportunistically switch and access unoccupied channels (called whitespaces and grayspaces) in spectrum bands originally licensed for other services on the condition of non-interference to the primary licensee or primary users. This provides an option to break away from the homogeneity of legacy spectrum access without the provider having to buy a fixed spectrum license. Empty channels 
within each band are liable to be selected for the actual data communication. To date, whitespace and grayspace networking has been allowed by policy in the TV Band [25, 30, 36], GSM Band [21], LTE Band [60, 62], and Citizen Broadband Radio Service (CBRS) Band [35] (Radar Band). While the original purpose of DSA was to improve spectrum efficiency [57] and reduce cross technology interference, we claim that the fact that it allows switching among different bands could be harnessed to improve the overall energy efficiency by intelligently matching a certain band (by virtue of its unique electro-magnetic properties) with different sensing modality requirements. While a decade-long body of DSA research focused on the interference free channel assignment given a particular band $[9,15]$, most of the energy-efficiency research has been limited to efficient channel sensing approaches only [1]. However, with wide-band spectrum analyzers and the notion of spectrum access systems (that coordinate spectrum assignments) [34] becoming increasingly available, soon a DSA end node is expected to have multiple choices for band selection.

\subsection{Contributions of This Paper}

In this article, we provide a thorough analysis of various aspects of wireless transmissions to identify the factors affecting the network energy efficiency, given various message requirements. In light of this analysis, we first propose a novel architecture for sustainable SCCs using a small-scale DSA overlay network on top of the existing legacy infrastructure. Next, we propose a dynamic band selection approach that exploits distinct electro-magnetic (EM) characteristics of different spectrum bands to intelligently match any message QoS and sensing modality requirements to a suitable band type for enhancing end-to-end energy efficiency while preserving the QoS. To this end, we formulate a linear optimization problem for determining an energy-efficient path for any message with hard deadline, termed as the HcE problem and prove that it is NP-Hard. Subsequently we present a pseudo-polynomial time dynamic programming (DP) algorithm for solving the problem exactly and thus determining the optimal HcE path (and thereby optimal band for transmission at each intermediate node) for such a message, provided that the DSA overlay topology is relatively steady. Additionally, to derive an efficient solution to the HcE problem, we propose a faster polynomial time greedy heuristic that combines both energy and latency costs to determine an optimal path for a certain message with hard deadline. Besides these, we investigate the dynamic selection approach under soft deadlines. In particular, we formulate the optimal path determination for a certain message with a soft deadline requirement, termed as the $S c E$ problem, as a non-linear optimization problem. Finally, we extend the proposed greedy heuristic to solve the ScE problem efficiently.

Through extensive simulation study, we evaluate the benefits of our proposed band selection approach in terms of both energy efficiency and QoS. Compared to the homogeneous band access and spectrum sharing approaches that are restricted to opportunistically access an available channel within a predetermined band, our study demonstrates that the dynamic wide-band selection approach greatly improves energy efficiency without sacrificing the QoS and incurring minimal infrastructure cost overhead. We further demonstrate that the proposed band selection approach works well for DSA overlay network in which the messages have soft deadlines. Finally, as a case study, we discuss some challenges of dynamic band selection for a variable DSA overlay topology, where a node may not have global knowledge of the topology.

The benefit of our novel work is that it provides an easy-to-deploy and low-cost option for designing green yet high-quality communication systems to realize non-urban SCCs. Moreover, for urban SCCs, it provides an alternative for improved energy-efficient communication network without burdening the wired infrastructures or incurring additional communication infrastructure cost. A preliminary version of this work appeared in ACM BuildSys 2017 [55]. 
In summary, this article makes the following important contributions:

- We propose a novel architecture for realizing SCCs using a small-scale DSA-enabled overlay network, on top of the traditional communication infrastructure.

- We propose a dynamic band selection approach that intelligently matches any message requirement to a suitable band type by exploiting distinct EM characteristics, such as operating frequency, and channel bandwidth of various bands.

- We formulate the determination of the best energy-efficient path for any message with hard deadline as a linear optimization problem, called the HcE problem, and prove it is NP-Hard.

- We present a pseudo-polynomial dynamic programming algorithm that solves the HcE problem exactly and determines the optimal HcE path for any message with hard deadline.

- We propose a faster polynomial time greedy heuristic that provides a fast and efficient solution to the HcE problem.

- We also investigate the proposed band selection approach under scenarios where messages have soft deadlines. Particularly, we formulate the determination of optimal path for a certain message with soft deadline, called the $S c E$ problem, as a non-linear programming-based optimization problem. Then, we extend our greedy heuristic for the HcE problem to the case of soft deadlines.

- We demonstrate through extensive simulation study that the proposed band selection approach outperforms homogeneous band access approaches in terms of both energy efficiency and QoS, while satisfying message deadline requirements, be it hard or soft.

- Finally, we highlight the challenges of a dynamic band selection approach in the case of a variable DSA overlay topology.

The rest of the article is organized as follows. Section 2 reviews the related work while Section 3 presents the proposed architecture. Section 4 discusses the distinct EM characteristics of various bands that can be exploited to achieve energy efficiency. Section 5 presents in detail the proposed band selection approach for steady DSA topology under hard deadlines. Section 6 investigates the proposed band selection under scenarios where messages have soft deadlines. Section 7 provides a discussion on cost-benefit analysis and improved sustainability for the proposed approach. Section 8 presents the simulation results, while Section 9 discusses challenges and road map for band selection under variable DSA topology, followed by conclusions in Section 10.

\section{RELATED WORK}

This section reviews the related literature for both traditional (non-DSA) and DSA/Spectrum sharing approaches for SCCs that use wireless cyber physical systems.

Several standard (non-DSA) wireless communication systems, e.g., DakNet [47] and KioskNet [54], have been proposed to provide connectivity by utilizing buses and public service vehicles (equipped with computers having WiFi radio) as mechanical backhaul to provide Internet connectivity to rural or remote communities. In contrast, JaldiMAC [7] utilizes long-range directional WiFi to form a wireless mesh network for providing connectivity in sparsely populated areas. Heimerl et al. [22] propose the deployment of low-power GSM-based Village Base Station (VBTS) for rural telephony. In LifeNet [32], the authors propose the connectivity utilizing hand-held wireless devices for transient conditions. In References [52, 53], the authors propose a four-tier hybrid architecture consisting of hand-held devices in the lowest tier, WiFi-enabled dropboxes in the second tier, data mules equipped with WiFi antennas in the third tier, and long-range WiFi towers in the fourth tier, thus providing connectivity in challenging scenarios such as post-disaster responses and remote areas. Google project Loon [39] introduces the network of balloons traveling 
on the edge of space, designed to extend Internet connectivity to people in rural and remote areas. Facebook Internet.org [38] utilizes local cellular infrastructure, along with satellite communications and UAVs, to deliver limited Internet connectivity to rural areas. However, all of these approaches utilize standard wireless technologies such as WiFi, WiMax, GSM base station, VSAT (very small aperture terminal), WLAN, wireless mesh, or a combination thereof, to provide connectivity to the rural communities. However, since all such devices access one same homogeneous band-usually industrial, scientific, and medical (ISM) or long-term evolution (LTE) or GSM bands-they suffer from under-provisioning or over-provisioning and policy constraints, as discussed later. Finally, third general partnership project (3GPP)/GSM technology providers are less motivated to invest in expensive wireless spectrum licenses in rural areas, even considering government subsidies [21].

Recently, the utilization of GSM whitespaces [21] and TV whitespaces [25, 30] have been proposed for rural and long-range connectivity, respectively. In Reference [21], the authors propose a hybrid sensing and database-driven spectrum sharing scheme, called Nomadic GSM, that utilizes GSM whitespaces for providing affordable rural connectivity. Nomadic GSM enables safe coexistence of primary and secondary users without requiring coordination or cooperation from existing license holders. The authors in Reference [25] propose utilizing TV band white space (TVWS) in conjunction with $5 \mathrm{G}$ infrastructure for Internet access in rural areas. In Reference [30], the authors propose opportunistic use of VHF/UHF TV bands through cognitive radio technology and DSA paradigm, such as IEEE 802.22 technology, for rural connectivity. Here the authors address two important issues that can affect the success of IEEE 802.22 technology in rural deployments. First, they propose suitable service model that combined TV broadcasting and data services to facilitate service adoption. Second, they propose an adaptive time division duplexing (TDD) approach to eliminate the requirement for long TDD turn-around time of existing IEEE 802.22 technology.

However, these approaches allow the secondary devices to opportunistically utilize the whitespaces in a pre-specified fixed band (e.g., TV band, GSM band, etc.) for data communication thus suffering from under-provisioning or over-provisioning. Additionally, such approaches require most sensing devices to be DSA enabled. In Reference [51], a cognitive wireless sensor network (CWSN) has been proposed for energy-efficient sensor network, where each sensor harnesses the DSA for energy efficiency. The authors primarily investigate the dynamic channel access problem to improve the energy efficiency of clustered CWSN. However, in such an approach, most sensor nodes have to support DSA functionalities, such as channel sensing and switching. Hence, the CWSN deployment at community scale is impractical as it requires every sensor to be DSA enabled, which is costly in terms of both end-to-end energy efficiency and monetary cost. In this article, we show that restricting the SCC data communications to only one such band is not optimally energy efficient given requirements of various sensing modalities.

\section{PROPOSED ARCHITECTURE}

In this section, we first present the key components of the proposed architecture for designing sustainable communication systems for SCCs (See Figure 1). Subsequently, we discuss the network model for DSA overlay network topology and the communication mechanism behind the proposed architecture.

\subsection{Key Components}

Sensor Block: A sensor block is a small physical subnetwork (e.g., a smart home, a smart metering infrastructure, an agriculture field, etc.) that comprises several heterogeneous sensing agents. A sensing agent could be a sensor (e.g., pollution sensor or temperature/light sensor) or a smart device (e.g., smart meter) that collects various sensory or contextual data in the form of text, image, 

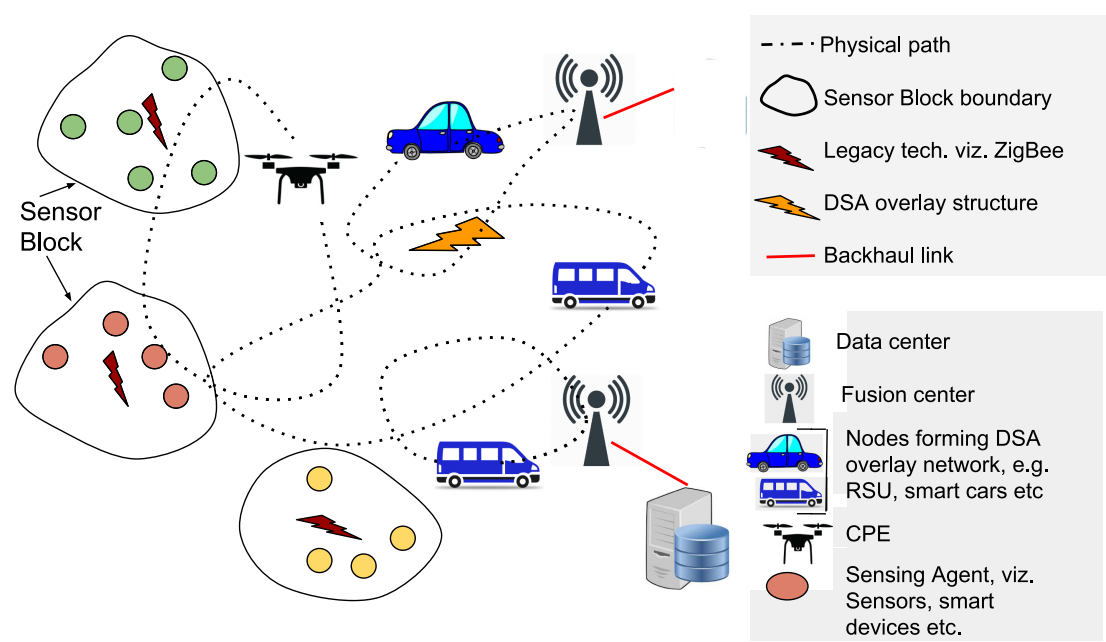

Fig. 1. Proposed communication architecture for SCCs.

audio, video, and so on. Such data may have different sizes, ranging from few KBs (text) to several MBs (image, video) and varying hard/soft delivery deadlines of the order of few hours (non-realtime deadlines). For example, a smart meter data usually has 0.5 - or 1-hour time deadlines for demand forecast, whereas data from pollution or moisture sensor may have larger deadlines (up to 5 to 6 hours). Therefore, each sensor block can be a source of heterogeneous data, each with varying demands. In addition, the distance between the sensor blocks and the data fusion center or the nearest backhaul link can have large variations, which we term geographical heterogeneity.

Owing to the smaller size of the sensor block, it can be assumed that the sensing agents within each block form a multi-hop ad-hoc network through low-power short-range legacy communication technologies that forward messages to a consumer premise equipment [14]. Unlike previous works [51], our proposed approach does not require sensing agents to be DSA enabled, thus making it practical and cost-effective.

Consumer Premise Equipment (CPE): A CPE acts as a proxy between sensor blocks and the DSA overlay network (explained in detail below). A CPE $q \in Q$, where $Q$ is the set of CPEs, may be a low-altitude flying drone or a moving vehicle that periodically collects various messages from a predetermined set of sensor blocks. This obviates the need for a dedicated infrastructure, such as separate access points or numerous DSA-enabled sensing agents, at each sensor block. We consider that each CPE has two wireless interfaces: (i) a low-power short-range wireless technology (e.g., Bluetooth or WiFi) to communicate with the sensing agents within the sensor block, and (ii) a DSA radio device (e.g., Ettus Research-N210 [37]) to communicate with other DSA-enabled devices (in the overlay network) on a certain band on a secondary basis (if they are in communication range over that band and there exists a free common channel).

Data Fusion Center: A data fusion center is a base station with a backhaul link to the final decision-making unit, where the generated messages from sensor blocks are delivered for computation and analytics purposes. The data fusion center is also equipped with a DSA radio device and possesses the capability to communicate with other DSA-enabled devices on a certain band (on a secondary basis).

DSA Overlay Network: The DSA overlay network acts as a bridge between the CPEs and the data fusion center. It comprises a set of cyber-physical components (the set of which is denoted by $R$ ), 


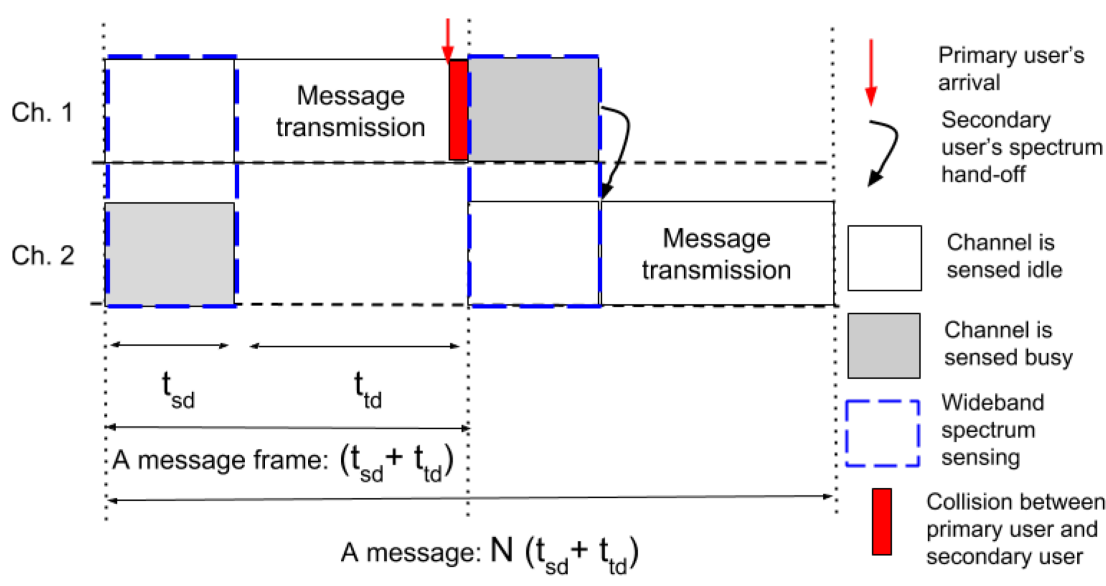

Fig. 2. A DSA node (or secondary user) message transmission-periodic sensing, message transmission, and spectrum hand-off.

each mounted with a DSA radio device. Examples include fixed road side assistance units (RSUs), connected public transportation, private smart vehicles, and so on. Such a DSA-enabled device opportunistically accesses an unoccupied channel on a suitable band for data communication. Hereafter in this article, a node refers to a physical component (e.g., flying drone, base station, public vehicle) mounted with a DSA radio device.

The specifics of discovery and selection of unoccupied (non-interfered) channel within a given band has been extensively discussed in a decade-long body of research [2, 9, 15]. Broadly, it can either be sense-and-use in the node (or DSA radio device) itself or through an off-device environmental sensing capability (ESC) [35]. In this article, we consider a sense-and-use mechanism for free channel selection in a given band, because it does not require any supporting framework of environmental sensing components. In such a mechanism [64], the message transmission is slotted via periodic sensing. Each periodic sensing frame consists of a sensing slot of duration $\left(t_{s d}\right)$ and a transmission slot of duration $\left(t_{t d}\right)$. Figure 2 shows the node's activities in transmitting a message. In the sensing slot, the node performs wide-band spectrum sensing to obtain the availability status of all the channels, whereas, in the transmission slot, the node sends the message packets over the chosen free channel. However, our proposed mechanism will also work seamlessly with an off-device environmental sensing capability(ESC) in a similar manner.

We assume that there exists $S$ set of bands where each band $s \in S$ has several (sub) channels. We have considered $S=\{$ TV, LTE, ISM, CBRS $\}$; however, it can easily be extended to any other unlicensed or licensed bands where DSA has been allowed. For example, millimeter-wave bands (30-300 GHz) are being discussed for communication in futuristic 5G technologies [40, 42]. The details of operating frequencies for aforementioned bands are shown in Table 1.

The DSA overlay network's topology could be of two types, which are as follows:

Steady Network Topology: In this case, the DSA overlay nodes are either fixed (e.g., RSUs) or have predictable mobility trajectories (e.g., public buses, municipal vehicles). Each node shares its geographical location and spectrum availability with other nodes in the network via a dedicated common control channel or other synchronization techniques as discussed in the recent DSA standards $[35,36]$. Hence, in such a steady topology, each node possesses global knowledge about approximate geographical location and spectrum availability at every other node. This steady DSA topology is the main focus of our article. 
Table 1. Spectrum Profile with Bandwidths

\begin{tabular}{|l|c|}
\hline Spectrum & Bandwidth \\
\hline $54-216 \mathrm{MHz}$ (VHF TV Band) & $6 \mathrm{MHz}$ \\
\hline $470-698 \mathrm{MHz}$ (UHF TV Band) & $6 \mathrm{MHz}$ \\
\hline $698-806 \mathrm{MHz}$ (700MHz Band/FirstNet) & $6 \mathrm{MHz}$ \\
\hline $700-800 \mathrm{MHz} / 1700-2100 \mathrm{MHz}$ (LTE Band) & $5-20 \mathrm{MHz}$ \\
\hline $902-928 \mathrm{MHz}$ (ISM Band) & $3-8 \mathrm{MHz}$ \\
\hline $2.4-2.5 \mathrm{GHz}$ (ISM Band) & $2-50 \mathrm{MHz}$ \\
\hline $3.5-3.7 \mathrm{GHz}$ (CBRS Band) & $10-40 \mathrm{MHz}$ \\
\hline $5.7-5.8 \mathrm{GHz}$ (ISM Band) & $20-80 \mathrm{MHz}$ \\
\hline
\end{tabular}

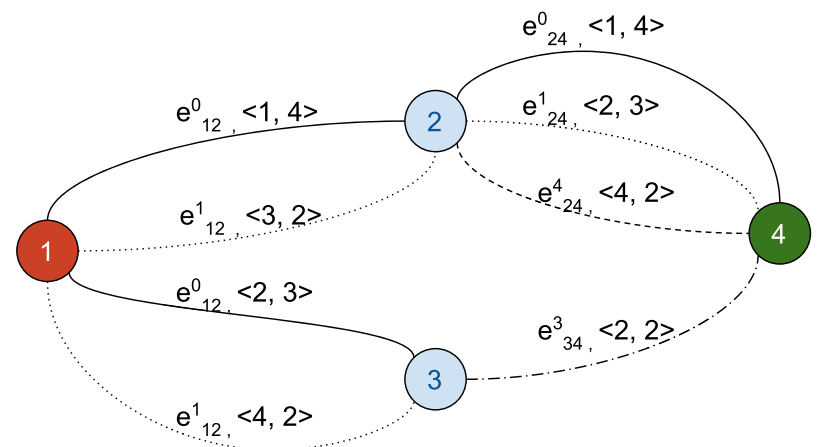

Fig. 3. Network model. $e_{12}^{0}$ and $e_{12}^{1}$ represent the links between node 1 and 2 over band type 0 and 1 , respectively. Moreover, the tuples $<1,4>$ and $<3,2>$ signify the energy and latency costs across those links, i.e., $e_{12}^{0}$ and $e_{12}^{1}$, respectively.

Variable Network Topology: In this case, the DSA overlay nodes may have variable (unpredictable) mobility pattern (e.g., a private vehicle), implying that a node may not possess accurate global knowledge of the network. However, it may gather local knowledge about the neighboring nodes via the common control channel.

\subsection{Network Model}

As illustrated in Figure 3, we model the DSA overlay network topology as a directed graph $G=$ $(V, S, E)$, where $V=Q \cup C \cup R$ is the set of all nodes including CPEs, data fusion centers, and cyber-physical devices in between; $S$ is the set of band types, and $E \subset(V \times V \times S)$ is the set of all directed links between two nodes over any common (free) channel in any band type. A link $e_{i j}^{(s)} \in E$ is a directed link from node $i$ to node $j$ over band $s \in S$. Hence, there may exist at most $|S|$ unique links between any node pair $i, j \in V$. A message traversing through $G$ is denoted by $m:\langle u, v, L, T\rangle$, where $u$ is the source, $v$ is the destination, $L$ is the message size, and $T$ is the hard delivery deadline. Now, for any given message $m$, each link is characterized by a tuple < $w_{i j}^{(s)}(L), \hat{t}_{i j}^{(s)}(L)>$, denoting the energy and latency costs, respectively. Note that this model is used for hard deadlines while soft deadlines are discussed in Section 6.

The energy cost $w_{i j}^{(s)}(L)$ refers to the total energy consumed in spectrum sensing and discovery, spectrum hand-off (or switching to an idle channel), and actual message transmission over a free channel in band $s$. We present the calculation of $w_{i j}^{(s)}(L)$ in Section 4.1. However, the latency 


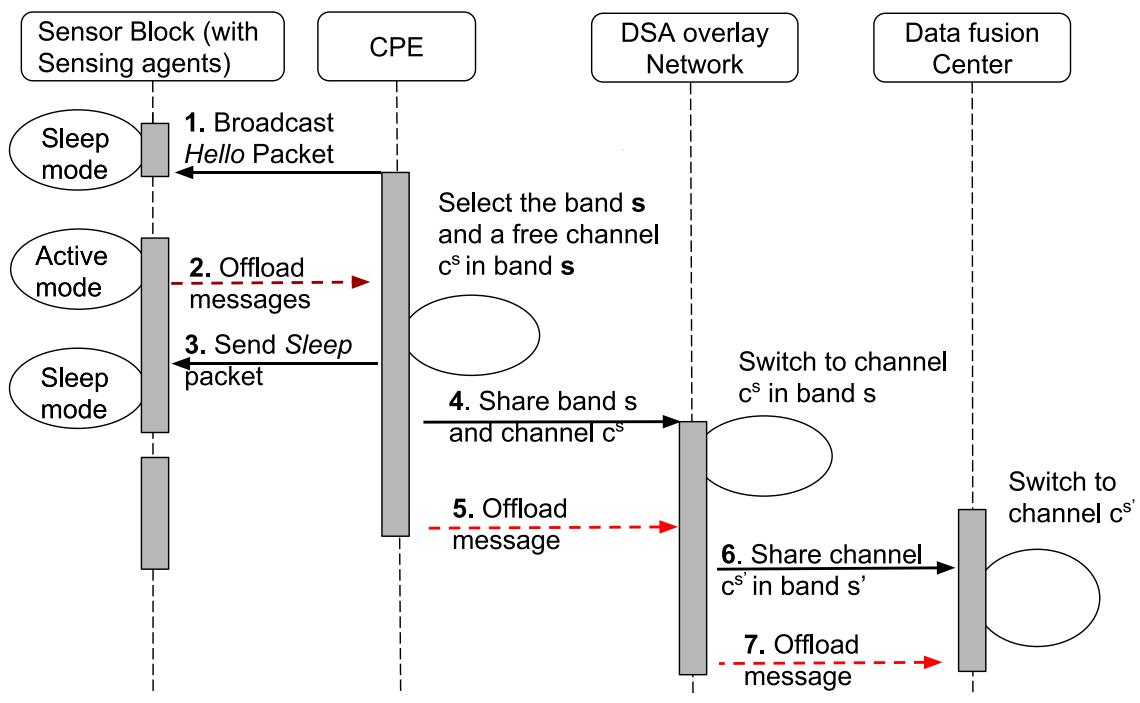

Fig. 4. Communication mechanism.

cost $\hat{t}_{i j}^{(s)}(L)$ denotes the total delay incurred due message transmission time (see Equation (3)), propagation time, and queuing delay (see Equation (9)).

\subsection{Communication Mechanism}

As shown in Figure 4, a CPE $q \in Q$ periodically broadcasts a Hello packet to the sensor blocks. On receiving the Hello packets, every sensing agent in a sensor block switches from a default energy-saving Sleep (receive only) mode to an Active (transmit and receive) mode and transmits the messages directly or indirectly via other sensing agents to the visiting $\mathrm{CPE}, q$. The $\mathrm{CPE}$ in turn broadcasts the Sleep packet in the sensor block such that the sensing agents switch back to the energy-saving Sleep mode. Meanwhile, the CPE computes the intermediate node and energyefficient band (say, $s \in S$ ) to transfer the messages to the data fusion center. For steady DSA topology, we discuss the band selection approaches (and determination of a set of intermediate nodes) for any message with hard and soft deadlines in Sections 5 and 6, respectively. We also touch on the band selection approach for variable DSA topology in Section 9.

Once the band $s$ is determined at CPE $q$, it shares the chosen band and channel (denoted by $c^{(s)}$ ) information with the intermediate node in the steady DSA network, which then switches to the channel $c^{(s)}$ in the chosen band $s$. Note that a unique intermediate node is determined in the steady topology, whereas in case of variable topology, $q$ may have to share the information with every neighboring nodes in the network such that the message delivery can be improved (see Section 9 for details). Next, the CPE $q$ transmits the message to the unique intermediate node (in steady DSA topology) and multiple neighboring nodes (in variable DSA topology) operating over the channel $c^{(s)}$ in the chosen band $s$. A similar process goes on at the intermediate/neighboring node(s) until the message is successfully offloaded to the data fusion center.

The rest of the article discusses how the proposed architecture (and communication mechanism) would enable dynamic band selection at each node and improve the network energy efficiency while guaranteeing QoS. To meet the objective of energy efficiency, it is evident that every message in the network needs to be offloaded to the data fusion center (or nearest backhaul link) in an energy-efficient manner and within the hard (or soft) deadline depending 
on the application requirement. Therefore, in this article, we primarily investigate the problem of determining energy-efficient path $p(u, v)$ for any given message $m$ from a source $u$ to a destination $v$, within a certain delivery deadline (be it hard or soft) such that the overall energy efficiency of the network is maximized yet preserving the QoS.

\section{TRADEOFFS BETWEEN BAND SELECTION AND ENERGY EFFICIENCY}

This section presents the energy consumption model for DSA overlay network. It also discusses how unique EM characteristics offered by various bands can be intelligently exploited to improve energy efficiency of the DSA overlay network, thereby realizing a green communication system for sustainable SCCs.

\subsection{Network Energy Consumption Model}

The DSA overlay network $G$ is influenced by various EM factors such as operating frequency, channel bandwidth, interference, and so on. Hence, the energy consumption model must consider all these factors into consideration. We discuss the relationship between energy consumption and various EM factors later in this section. Now, given a path $p(u, v)$ for a certain message $m$ (with size $L$ ) from a source node $u$ to destination node $v$, the total consumed energy is given by

$$
w_{u v}(L)=\sum_{e_{i j}^{(s)} \in p(u, v)} w_{i j}^{s}(L),
$$

where $w_{i j}^{(s)}(L)$ is the energy cost over link $e_{i j}^{(s)}$ and $e_{i j}^{(s)}$ is an intermediate link in path $p(u, v)$.

Calculation of Energy Cost $w_{i j}^{(s)}(L)$ : As discussed earlier in Section 3, each node determines a free (unoccupied) channel via sense and use mechanism. Under this consideration, similarly to Reference [64], the energy cost $w_{i j}^{(s)}(L)$ over a link $e_{i j}^{(s)}$ can be expressed as

$$
w_{i j}^{(s)}(L)=N t_{s d} \tilde{P}+N \mathcal{P} w_{s w}+T_{t d} P_{i}^{(s)}
$$

where $N$ is the number of time slots required for one message transmission and can be calculated as the fraction of message size $(L)$ to the effective bit rate $\left(\mathbb{R}_{i j}^{(s)}\right)$ of a certain band $s$, i.e., $\left\lceil\frac{L}{\mathbb{R}_{i j}^{(s)}}\right\rceil$. In addition, $t_{s d}$ is the sensing slot duration; $\tilde{P}$ is the spectrum sensing power (in watts); $\mathcal{P}$ is the probability of switching to an idle channel, termed as spectrum hand-off; $w_{s w}$ is the energy cost (in Joules) of one channel switching; $P_{i}^{(s)}$ is the transmit power at node $i$ over band $s$. Finally, $T_{t d}$ is the average time required to transmit the entire message and can be computed as $T_{t d}=N \mathcal{P} t_{t d}$, where $t_{t d}$ denotes the transmission slot duration (see Figure 2).

For each message frame, the DSA node has to sense for $t_{s d}$, and $N$ frames are required for one message transmission. Hence the energy consumed in sensing is $N t_{s d} \tilde{P}$. Given that the node switches to another channel with probability $\mathcal{P}$, it consumes $N \mathcal{P} w_{s w}$ amount of energy in channel switching. The last part of Equation (2) indicates the total energy consumed in transmitting message of size $L$ over a channel in band $s$. Usually, $t_{s d}$ and $t_{t d}$ are pre-determined values for any given band type, and $w_{s w}$ is negligible. Thus, $N=\left\lceil\frac{L}{\mathbb{R}_{i j}^{(s)}}\right\rceil$ and $P_{i}^{(s)}$ are the two main contributing factors for the energy cost of any link.

Given $t_{s d}, t_{t d}, N$, and $\mathcal{P}$, the total message transmission time, denoted by $t_{i j}^{(s)}(L)$, for a message $m$ can be calculated as follows:

$$
t_{i j}^{(s)}(L)=N\left(t_{s d}+\mathcal{P} t_{t d}\right)=\left\lceil\frac{L}{\mathbb{R}_{i j}^{(s)}}\right\rceil\left(t_{s d}+\mathcal{P} t_{t d}\right) .
$$


From Equations (1), (2), and (3), it is clear that the total consumed energy is directly proportional to the transmit power, the number of transmissions, and message transmission time. Based on these observations, we discuss the impact of different EM characteristics offered by various bands on the overall energy efficiency of the DSA overlay network.

\subsection{Operating Frequency vs. Energy Efficiency}

It is known that the received signal strength at a receiver must meet some reception threshold, $\tau$, to be able to decode a signal accurately. The generalized Frii's transmission equation [31] provides the relationship between the received power at receiver $j$ and the transmit power from transmitter $i$ over any channel with a representative frequency ${ }^{1} f^{(s)}$ in band $s$,

$$
P_{j}^{(s)}=P_{i}^{(s)} G_{i} G_{j}\left(\frac{\mathbb{C}}{4 \pi f^{(s)} d}\right)^{\alpha} \geq \tau,
$$

where $G_{i}$ and $G_{j}$ are the transmitter and receiver antenna gains, $\alpha$ is path loss exponent, $\mathbb{C}$ is the speed of light, $d$ is the distance between $i$ and $j, P_{i}^{(s)}$ is the transmit power of $i$, and $P_{j}^{(s)}$ is the received power at $j$. Now let $\phi=G_{i} G_{j}\left(\frac{\mathbb{C}}{4 \pi}\right)^{\alpha}$ be a constant.

Thus the above equation becomes $P_{j}^{(s)}=\phi \frac{P_{i}^{(s)}}{\left(f^{(s)} d\right)^{\alpha}}$. It is evident that for higher frequencies, the transmit power $P_{i}^{(s)}$ ought to be increased to maintain the same $P_{j}^{(s)} \geq \tau$, even though the distance $d$ is unchanged. The resultant increased transmit power causes more energy consumption (refer to Equations (1) and (2)). Alternatively, if the transmit power is not increased, then $\tau$ is only met at a lower distance. Hence, more intermediate hops would be required to traverse the same distance $d$, leading to the additional transmission and reception operations at each intermediate hop that causes higher energy expenditure.

Moreover, the lower frequencies have larger wavelengths yielding better obstacle and wall penetration capabilities. This implies the lower frequencies are less error prone, thus reducing (re)transmission overheads and enabling better non-line-of-sight connectivity. Among the bands that allow DSA, the TV and LTE bands have lower frequency allocations than traditionally used unlicensed band of $2.4 \mathrm{GHz}$. In other words, these bands should be seemingly more energy efficient.

However, lower frequencies come at a tradeoff in the sense that they usually have much lesser bandwidth of $6 \mathrm{MHz}$ as opposed to the LTE (upto 20MHz) and CBRS (upto 40MHz). For larger message sizes, the message transmission time (refer to Equation (3)) will be higher as the number of time slots $(N)$ increases. Supporting high data rate applications are also challenging for lower frequencies offering smaller bandwidths. However, frequencies offering higher bandwidths will reduce the transmission time for a given message size, thereby saving energy. An important research question, therefore, is whether the reduction in the transmission time for higher frequencies offsets the gain in energy efficiency due to the reduced hop count.

\subsection{Bandwidth vs. Energy Efficiency}

The relationship between the bandwidth and the dissipated power could be understood through Rayleigh-Parseval Equation [12]:

$$
P_{d i s}^{(s)}=\int_{f_{1}^{(s)}}^{f_{2}^{(s)}} \mathbb{S}_{i}\left(f^{(s)}\right) d f,
$$

\footnotetext{
${ }^{1}$ For example, $f_{S}=2.4 \mathrm{GHz}$ for the frequencies in the range $2.412-2.462 \mathrm{GHz}$ used by legacy WiFi devices in ISM band.
} 
where $\mathbb{S}_{i}$ is the power spectral density, $\Delta f^{(s)}=f_{2}^{(s)}-f_{1}^{(s)}$ is the bandwidth of any channel in the band type $s$, and $P_{d i s}^{(s)}$ is the total power dissipated over band $s$. From Equation (5), it is clear that a channel offering higher bandwidth causes larger power dissipation. Given a fixed transmission time for any message, the higher bandwidth channels will consume more energy than the lower ones. The typical bandwidths for various spectrum is listed in Table 1.

Conversely, from Shannon-Hartley theorem [56], the effective bit rate $\left(\mathbb{R}_{i j}^{(s)}\right)$ between a transmitter $i$ and receiver $j$ over any channel in band $s$ with representative frequency $f^{(s)}$ is given by

$$
\mathbb{R}_{i j}^{(s)}=B^{(s)} \log _{2}\left(1+\chi_{i j}^{(s)}\right)=B^{(s)} \log _{2}\left(1+\phi \frac{P_{i}^{(s)}}{\left(f^{(s)} d\right)^{\alpha} N^{(s)}}\right),
$$

where $B^{(s)}$ is the bandwidth of any channel in band type $s$ and $\chi_{i j}^{(s)}=\frac{P_{j}^{(s)}}{N^{(s)}}$ is the ratio of the communication signal power to the interference and noise at the receiver (SINR). The rest of the notations are the same as above. From Equation (6), given that the SINR is unchanged, a higher bandwidth channel would offer a higher effective bit rate, thereby decreasing the required transmission time.

Therefore, from the above relationships of bandwidth with (i) dissipated transmit power (Equation (5)) and (ii) effective bit rate (Equation (6)), the higher the channel bandwidth, the higher is the dissipated power but the lower is the transmission time. Thus, the tradeoff is whether to choose a high bandwidth channel that reduces the transmission time and increases the dissipated power or vice versa.

\subsection{Interference and Packet Size vs. Energy Efficiency}

Increased cross device interference will increase the noise floor as more and more devices coexist in the unlicensed spectrum $(2.4 \mathrm{GHz})$ and GSM/LTE $(1700-2100 \mathrm{MHz})$ band. Therefore, from Equation (6), it is evident that the SINR will decrease. To maintain a certain required SINR, the transmit power needs to be increased. However, switching to any other band type using DSA would allow us to maintain the same SINR with a low transmit power, thus saving energy.

Packet sizes in the existing low-power short-range technologies $(2.4 \mathrm{GHz})$ are usually small (maximum size 128 bytes), because the bit error rate is very high. However, communication through lower frequency bands experiences smaller bit error rate (as explained in Section 4.2). Thus, as shown in Reference [29], larger packet sizes can be employed that would reduce perpacket overheads such as headers and preambles, and therefore, promises energy efficiency.

\section{BAND SELECTION UNDER HARD DEADLINES}

In this section, we discuss the proposed band selection approach for steady DSA network topology where messages have hard delivery deadline requirements. In such a topology (see Section 3), each node possesses global knowledge about the approximate geographical location and spectrum availability at every other node in the network. The key idea of the band selection approach under hard deadlines is to intelligently match each message $m:<u, v, L, T>$ to a suitable band at each intermediate node between the source node $u$ and the destination node $v$ so as to construct an optimal hard deadline constrained energy-efficient $(\mathrm{HcE})$ path $p(u, v)$. We first formulate the $\mathrm{HcE}$ path determination as a linear optimization problem and show that it is NP-hard. Then, we present a pseudo-polynomial dynamic programming approach that selects the best band at each intermediate node, for constructing an optimal HcE path for any given message. Following it, we propose a faster (though not optimal) polynomial-time greedy heuristic for solving the HcE problem. 


\subsection{HcE Problem Formulation}

As mentioned, the $\mathrm{HcE}$ path problem aims at determining the most energy-efficient path for any given message that also meets the hard delivery deadline. Although we solve the optimization problem for a given message $m$, it will also work well for concurrent messages. This is because a band is composed of several channels that can be simultaneously accessed by concurrent messages (should they end up choosing the same band in the same geographical region), unless in the unlikely rare event where the number of such messages exceed the total number of available channels. There may be a small channel access delay due to channel bargaining within the selected band, which is negligible in the range of micro seconds [11] and hence is ignored in the optimization formulation.

Given a directed graph $G=(V, S, E)$, a source-destination node-pair $u, v \in V$, a message $m:<$ $u, v, L, T>$, each link $e_{i j}^{(s)} \in E$ characterized by energy cost $w_{i j}^{(s)}(L)$ and latency cost $\hat{t}_{i j}^{(s)}$, the HcE path problem can be formally defined as

$$
\min _{p(u, v) \in P^{\prime}(u, v)} \sum_{e_{i j}^{(s)} \in p(u, v)} w_{i j}^{(s)}(L),
$$

where $P^{\prime}(u, v) \subset P(u, v)$ is the set of all paths from the source node $u$ to the destination node $v$, which meets the following five constraints:

(i). SINR constraint. For successful decoding of any message $m$ at receiver $j$, the achieved SINR $\chi_{i j}^{(s)}$ at receiver $j$ must be greater than or equal to prespecified SINR threshold $\chi_{t h}$, typically a small value, say, $-75 \mathrm{dBm}$.

(ii). Power constraint. As per the federal communications commission (FCC) guidelines, the maximum transmit power, $P_{i}^{(s)}$ at any transmitter $i$ must be less than or equal to a fixed value, the maximum effective isotropically radiated power (EIRP), $P_{\max }^{(s)}$ over any chosen band $s \in S$.

(iii). Transmission coverage constraint. For successful data communication over any intermediate link $e_{i j}^{(s)} \in p$, the Euclidean distance $d_{i j}$ must be less than or equal to the transmission coverage $\gamma^{(s)}$ achieved in the chosen band $s$, where $\gamma^{(s)}=\left[G_{i} G_{j}\left(\frac{c}{4 \pi f^{s}}\right)^{\alpha} \frac{\tau}{P_{j}^{s}}\right]^{\frac{1}{\alpha}}$ (refer to Frii's transmission equation Equation (4) for details).

(iv). Unique spectrum constraint. Any transmitter-receiver node pair $i, j \in V$ must tune to a common available channel in a unique spectrum band $s \in S$. That is, though there are potentially $|S|$ possible links between nodes $i$ and $j$, only one link over a certain band can be utilized at any point of time (due to hardware constraints),

$$
\sum_{s \in S} e_{i j}^{(s)} \leq 1, \quad \forall e_{i j}^{(s)} \in p
$$

(v). Hard delivery deadline constraint. Any message must be delivered within the hard deadline from its source node $u$ to the destination $v$. Hence, as shown in Equation (9), the latency cost $\hat{t}_{i j}^{(s)}(L)$ i.e., the sum of message transmission time $t_{i j}^{(s)}(L)$, propagation time $\frac{d_{i j}}{\mathbb{C}}$, and queuing time $\left(q_{\epsilon}\right)$ over each intermediate link in the path $p(u, v)$, must be less than or equal to the hard deadline $T$. Thus,

$$
\sum_{e_{i j}^{(s)} \in p(u, v)} \hat{t}_{i j}^{(s)}(L)=\sum_{e_{i j}^{(s)} \in p(u, v)} t_{i j}^{(s)}(L)+\frac{d_{i j}}{\mathbb{C}}+q_{\epsilon} \leq T,
$$


where $\mathbb{C}$ is the propagation speed of any spectrum (usually speed of the light). The propagation time $\left(\frac{d_{i j}}{\mathbb{C}}\right)$ and queuing time $\left(q_{\epsilon}\right)$ are usually negligible compared to the message transmission time $t_{i j}^{(s)}(L)$. In the generalized term, the total of latency cost is equivalent to the total message transmission time, i.e., $\sum_{e_{i j}^{(s)} \in p(u, v)} \hat{t}_{i j}^{(s)}(L)=\sum_{e_{i j}^{(s)} \in p(u, v)} t_{i j}^{(s)}(L) \leq T$.

\section{Theorem 5.1. The HcE path determination problem is NP-Hard.}

Proof. We provide a reduction from the Restricted Shortest Path (RSP) problem [27, 67]. Let us consider a generic instance of the RSP problem. Consider a graph $G_{r s p}\left(V_{r s p}, E_{r s p}\right)$, where $V_{r s p}$ is the set of nodes and $E_{r s p} \subseteq\left(V_{r s p} \times V_{r s p}\right)$ is the set of edges. Each edge $e_{i j} \in E_{r s p}$ is associated with a cost $c_{i j}$ and an additional metric $t_{i j}$. Then, given a source node $u$, a destination node $v$, and constraint bound $D$, the goal of the RSP problem is to determine the least cost path $p_{r s p}(u, v)$ such that $\sum_{e_{i j} \in p_{r s p}(u, v)} t_{i j} \leq D$.

We reduce this RSP problem to an instance of the HcE problem as follows. Consider a DSA network topology graph $G(V, S, E)$ where a node in $V$ is created for each node in $V_{r s p}$. Assume that $S$ contains a single band and an edge is added to $E$ for each edge in $E_{r s p}$. In addition, for each edge $e_{i j}$ we set $w_{i j}^{(1)}(L)=c_{i j}$ and $\hat{t}_{i j}^{(1)}(L)=t_{i j}$. Finally, we set the hard deadline $D=T$.

Given such an instance, the HcE problem determines the least energy cost path $p(u, v)$ that also meets the hard deadline $T$. Such path also corresponds to an optimal solution of the RSP problem, since it is the shortest cost path satisfying the constraint $D$. Therefore, if we were able to solve $\mathrm{HcE}$ problem in polynomial time, the RSP problem will also be solvable in polynomial time. Since RSP is NP-Complete, we conclude that the HcE problem is NP-Hard.

\subsection{Dynamic Programming Approach}

In the following we present an exact pseudo-polynomial DP algorithm for solving the HcE optimization problem. The DP algorithm intelligently matches a message $m$ to an optimal (energyefficient) band such that the total energy cost incurred in path $p(u, v)$ is minimized while meeting the hard deadline and other constraints. The proposed DP algorithm is a combination of the Floyd-Warshall algorithm for all pair shortest paths and the dynamic programming solution of the Knapsack problem [13].

Now, for a message $m$ with $L$ bits and a hard deadline $T$, the graph $G(V, S, E)$ can be represented by a 3D adjacency matrix $A:|V| \times|V| \times|S|$. An element of $A[i, j, s]$ is an ordered pair $<w_{i j}^{(s)}(L), \hat{t}_{i j}^{(s)}(L)>$ where the first and second parts denote the energy and latency costs, respectively, for a message $m$ along the link $e_{i j}^{s}$ from node $i$ to node $j$ over band $s \in S$.

Here,

$$
w_{i j}^{(s)}(L)= \begin{cases}0, & i=j \\ w_{i j}^{(s)}(L), & i \neq j, \text { and first four constraints in Section } 5.1 \text { are met } . \\ \infty & \text { otherwise }\end{cases}
$$

Similarly, $\hat{t}_{i j}^{(s)}(L)$ can be defined.

The proposed DP algorithm is based on the notion of intermediate node, defined as follows:

Definition 1. (Intermediate node): Given a path $p=<1,2, \ldots,(|l|-1),|l|>$, where nodes in the path are represented as integers, an intermediate node $i$ is one such as $2 \leq i \leq|l|-1$.

The DP algorithm is based on the following observation. Consider a subset of nodes $\{1, \ldots k\}$. For any node pair $i, j \in V$, let $p$ be the path with the least energy cost, out of all paths between $i$ and $j$ whose intermediate nodes are in $\{1, \ldots k\}$. Then, there are two possible cases. 


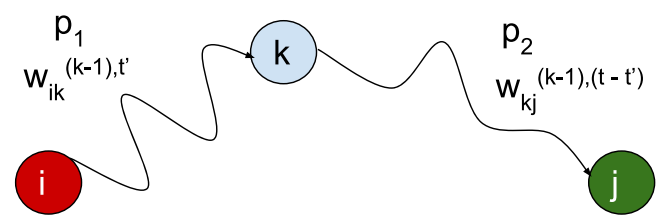

Fig. 5. Path $\mathrm{p}$ is the energy-efficient path from node $i$ to node $j$ with deadline $t$, and $k$ is the highest numbered intermediate node of path $p$. Sub-path $p_{1}$ from node $i$ to $j$, has all the intermediate nodes in $\{1,2, \ldots k-1\}$ and the hard deadline is $t^{\prime}$. Similarly, sub-path $p_{2}$ from node $k$ to $j$, has all intermediate nodes in $\{1,2, \ldots k-1\}$ and hard deadline $\left(t-t^{\prime}\right)$.

(a) $k$ is not an intermediate node of $p$ : In this case, all the intermediate nodes of path $p$ are in $\{1, \ldots, k-1\}$. Hence, the energy-efficient path from $i$ to $j$ with intermediate nodes $\{1, \ldots, k\}$ is also the energy-efficient path from $i$ to $j$ with intermediate nodes in $\{1, \ldots, k-1\}$.

(b) $k$ is an intermediate node of $p$ : In this case, $p$ can be broken into two sub-paths: $p_{1}$ from $i$ to $k$ and $p_{2}$ from $k$ to $j$. Since $p$ is the energy-efficient path, $k$ can only appear once in $p$. Therefore, $k$ can not be an intermediate node of $p_{1}$ or $p_{2}$. Moreover, by the principle of optimality (that states that the sub-paths of a shortest path are also shortest paths), both $p_{1}$ and $p_{2}$ are the shortest, i.e., energy-efficient paths between $i$ and $k$, and $k$ and $j$, respectively, with intermediate nodes in the set $\{1, \ldots k-1\}$. Figure 5 provides an illustration.

Let us now define the subproblems used by the DP approach that uses the intermediate nodes used and satisfies the hard deadline. Let $w_{i j}^{(k, T)}$ be the energy cost of the energy-efficient path from node $i$ to $j$ that meets hard deadline $T$ and uses only intermediate nodes in the set $\{1,2, \ldots, k\}$. The solutions of subproblems can be formulated recursively as follows, leading to the solution of the overall problem.

(1) For $T=0$, there is no path, hence $w_{i j}^{(k, 0)}=\infty$, for each $i, j \in V$.

(2) For $k=0$, there are no intermediate nodes in the path, hence for each $i, j \in V$,

$$
w_{i j}^{(0, T)}=\left\{\begin{array}{ll}
\min _{s \in S} & w_{i j}^{(s)}(L), \text { if } \exists s \in S \text { such that } \hat{t}_{i j}^{(s)}(L) \leq T . \\
\infty & \text { otherwise }
\end{array} .\right.
$$

(3) For $k>0$ and $T>0$, we can select a path with the least energy cost either using $k$ as the intermediate node or not. If $k$ is used, then let $t_{1}$ be the delay over the sub-path between $i$ and $k$, and $t_{2}$ the delay for the sub-path between $k$ and $j$. To ensure that the overall path meets the deadline $T$, we need to find the value of $t \in[1, \ldots, T]$ such that if given $t_{1}$, then $t_{2}=t-t_{1}$. Formally,

$$
w_{i j}^{(k, T)}=\min _{t=1 \ldots T}\left(w_{i j}^{(k-1), t}, \min _{t_{1}=0 \ldots t}\left(w_{i k}^{(k-1), t_{1}}+w_{k j}^{(k-1),\left(t-t_{1}\right)}\right)\right) .
$$

When $k=|V|$ and $t=T$, we obtain the final solution, which is the total energy cost for the energy-efficient path for each pair of source-destination node-pair with hard deadline $T$. In other words, $W^{(|V|, T)}=\left(w_{i j}^{(|V|, T)}\right)$ for all $i, j \in V$.

5.2.1 DP Algorithm Description. Algorithm 1 uses a series of 3D matrices $W^{(k, t)}$ for $k=$ $0, \ldots|V|$ and $t=0, \ldots T$. The matrix $W^{(k, t)}$ contains the elements $w_{i j}^{(k, t)}$, that is the hard deadline $(1 \leq t \leq T)$ constrained energy-efficient path between $i$ and $j$ using the intermediate nodes in $\{1, \ldots k\}$. Also, we keep track of the optimal HcE path in another matrix $P_{i j}^{(k, t)}$. The algorithm has the following two steps: 


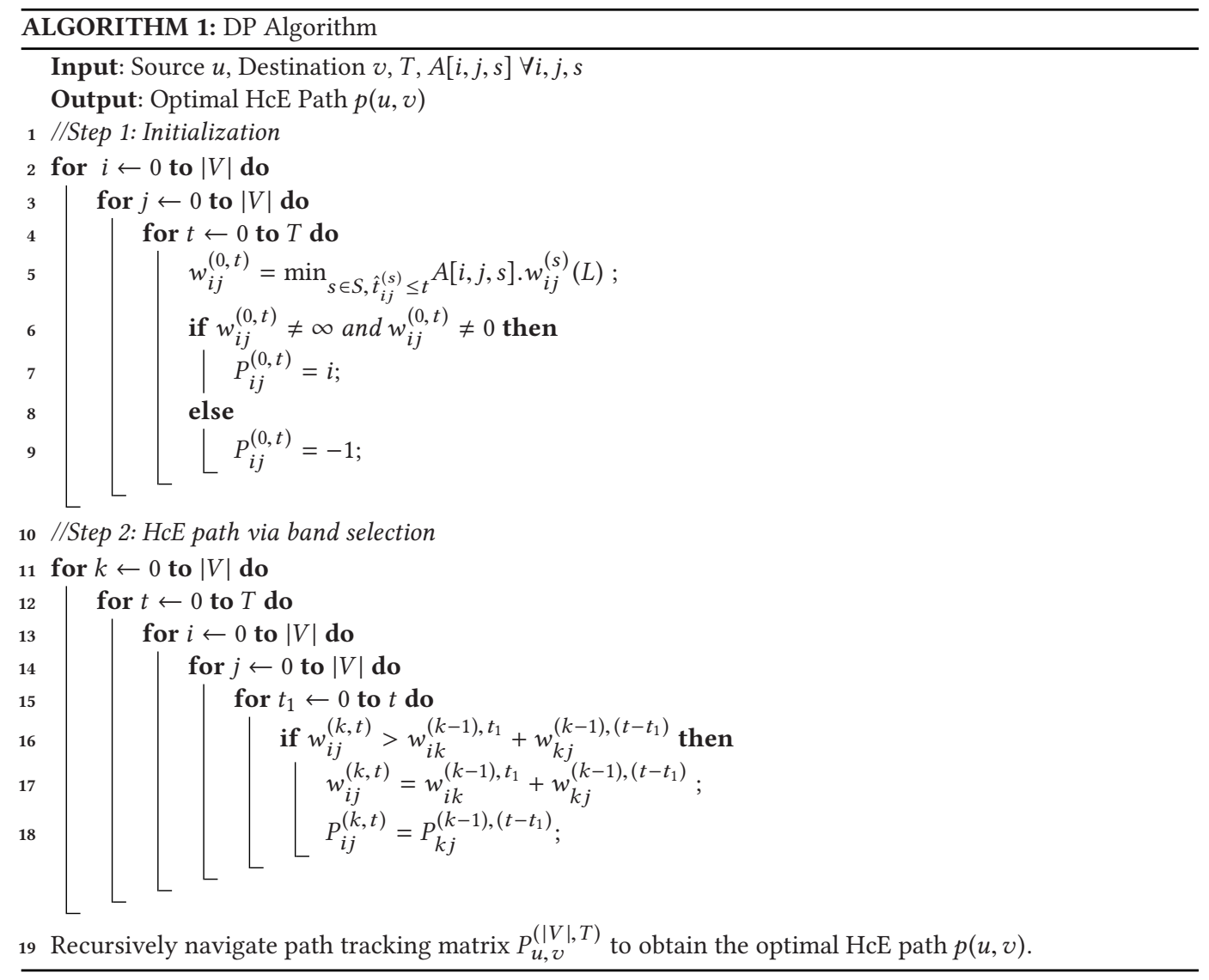

Step 1 (Initialization): The algorithm first initializes the matrix $W^{(0, T)}$ to $A[i, j, s]$. The HcE path tracking matrix, $P^{(0, T)}$ is also initialized in lines 2-9 (see Equation (13)),

$$
w_{i j}^{(0, t)}=\left\{\begin{array}{l}
0 \quad \text { if } i=j \\
\min \left(w_{i j}^{(0, t-1)}, \min _{s \in S} w_{i j}^{(s)}(L)\right) \text { if } i \neq j \text { and } \hat{t}_{i j}^{(s)}(L)=t . \\
\infty \quad \text { otherwise }
\end{array}\right.
$$

Step 2 (HcE path via band selection): After the initialization, the algorithm applies the recursive formula to calculate $W^{(k, t)}$ given $W^{\left(k-1, t_{1}\right)}$ and $W^{\left(k-1, t-t_{1}\right)}$, where $1 \leq t_{1} \leq t$, as shown in Algorithm 1. Also, the path tracking matrix $P$ is updated recursively (Lines 11-19).

Finally, any source node $u$ delivers the message $m(u, v, L, T)$ from a source $u$ to the destination $v$ via the computed optimal HcE path $p(u, v)$ obtained from the path tracking matrix $P$.

Time Complexity: From the description of Algorithm 1, the time complexity of Step 1 (lines 2 - 9) is $O\left(|V|^{2} T\right)$, whereas the time complexity of Step 2 (lines 11-18) is $O\left(|V|^{3} T^{2}+|V|\right.$ ), where $|\mathrm{V}|$ is the number of nodes and $T$ is the hard deadline. Thus, the overall time complexity of the DP algorithm is $O\left(|V|^{3} T^{2}\right)$, which is not polynomial in the input size, since it also depends on the value of $T$. Such time complexity is referred to as pseudo-polynomial. 


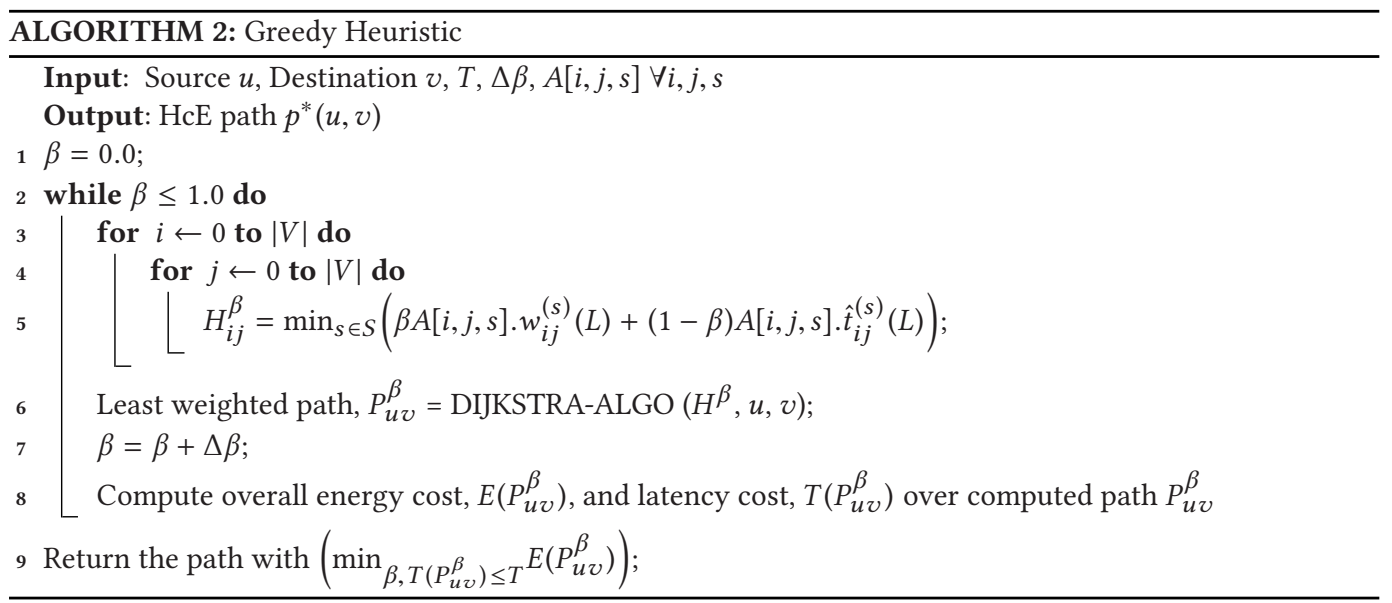

\subsection{HcE Greedy Heuristic}

This section presents a polynomial time greedy heuristic for an approximate solution of the HcE path $p^{*}(u, v)$ for a given message in the network. The underlying idea is to calculate a weight $h_{i j}$ for each edge $e_{i j} \in E$ that combines the energy cost $w_{i j}^{(s)}(L)$ with the latency cost $\hat{t}_{i j}^{(s)}(L)$. A parameter $\beta \in[0,1]$ defines the weighted average between the two, such that $h_{i j}=\beta \times w_{i j}^{(s)}(L)+(1-\beta) \times$ $\hat{t}_{i j}^{(s)}(L)$. Intuitively, using Dijkstra algorithm [13] and setting $\beta=0$, we can use such weights to calculate the minimum delay path, which may not necessarily be energy efficient. Similarly, if we set $\beta=1$, then we can calculate the most energy efficient path, which may not meet the deadline $T$. By increasing $\beta$ from 0 to 1 in steps of $\Delta \beta$, we can find a path that tradeoffs energy efficiency and delay.

The pseudo code of the proposed greedy heuristic is presented in Algorithm 2. Given a value of $\beta$ the proposed heuristic computes the weighted metric $h_{i j}$ for each node pair $i, j$ that forms the matrix $H_{i j}^{\beta}$ as follows (see line 5):

$$
H_{i j}^{\beta}=\beta w_{i j}^{(s)}(L)+(1-\beta) \hat{t}_{i j}^{(s)}(L)
$$

The heuristic then calls Dijkstra's algorithm to compute the least weighted path $P_{u v}^{\beta}$ from source $u$ to the destination $v$ (line 6) using the values of $H_{i j}^{\beta}$ as edge weights. Then, it computes the total energy cost denoted by $E\left(P_{u v}^{\beta}\right)$ and the total latency cost denoted by $T\left(P_{u v}^{\beta}\right)$ to compute the least weighted path $P_{u v}^{\beta}$. The above steps (lines 2-6) are iterated for all values of $\beta$ in the increased interval of $\Delta \beta$. Finally, the $\operatorname{HcE}$ path $p^{*}(u, v)$ is chosen as the path with minimum $E\left(P_{u v}^{\beta}\right), \quad \forall \beta$ such that $T\left(P_{u v}^{\beta}\right) \leq T$.

Time Complexity: The time complexity of Algorithm 2 is $O\left(\frac{1}{\Delta \beta}|V|^{2}+\frac{1}{\Delta \beta}|V| \log (|V|)+\frac{1}{\Delta \beta}\right)$, since the algorithm performs $O\left(\frac{1}{\Delta \beta}\right)$ iterations and it takes $O\left(|V|^{2}\right)$ time to compute $H_{i j}^{\beta}, \forall i, j \in V$. Similarly, it takes $O(|V| \log (|V|))$ time to run Dijkstra's algorithm and $O(1)$ time to compute the minimum $E\left(P_{u v}^{\beta}\right), \forall \beta$. Hence, the time complexity of the greedy heuristic is $O\left(\frac{1}{\Delta \beta}|V|^{2}\right)$.

The following theorem proves the non-trivial property that the heuristic always returns a solution, if one exists. In other words, the greedy choices of the algorithm, although potentially not optimal, do not prevent us from finding a path that meets the hard delivery deadline, $T$. 
THEOREM 5.2. Provided that there exists at least a HcE path $p^{\prime}(u, v)$ for a message $m$ with size $L$ and hard deadline $T$ in the DSA overlay network, the greedy heuristic will always find such a solution.

Proof. Consider the weight parameter $\beta=0$ at the first iteration of the heuristic. Then, the weight metric $H_{i j}^{\beta}$ is given by $H_{i j}^{\beta}=\hat{t}_{i j}^{(s)}(L), \forall i, j \in V$. This implies the latency cost of each link $e_{i j}^{(s)}$ for a message $m$ is used as the weight. By executing Dijkstra's algorithm with these weights, the path between $u$ and $v$ with minimum latency can be obtained. If such a path meets the deadline $T$, then this becomes a more energy-efficient path. Otherwise, there exists no solution, since all the paths found in the subsequent iterations will not also meet the deadline. This also implies that such path iss the shortest in terms of latency.

\section{BAND SELECTION UNDER SOFT DEADLINES}

In this section, we extend our dynamic band selection approach for the steady DSA overlay topology to the case in which messages have soft delivery deadlines. Soft deadlines have been largely investigated in the context of real-time systems and scheduling theory $[3,16,26,28,48,68]$. In general, a soft deadline allows a certain message to be delivered after a desired delivery deadline, however by incurring a penalty defined by the penalty function. Using the commonly adopted exponential penalty function, we formulate a non-linear programming (NLP) optimization problem, termed the $S c E$ problem, to determine an optimal energy-efficient path under soft deadlines. Since NLP is hard to solve practically [6], we extend our proposed greedy heuristic for HcE to solving the ScE problem.

\subsection{Penalty Function}

The penalty function increases as the delay in delivering a certain message increases. Thus, the penalty is a non-negative function of message delivery time. There exist several possible definitions of soft deadline requirements, such as: (i) no more than X consecutive deadlines can be missed, (ii) no more than $\mathrm{X}$ deadlines in an interval of time $T$ can be missed, (iii) the deadline miss ratio (percentage or total missed deadlines over the total number of deadlines) must not exceed a certain threshold, and (iv) the penalty of delivering a message is zero before a desired deadline $T_{d}$; it increases according to a prespecified penalty function up to a maximum tardiness $T_{\max }$, after which the penalty becomes infinity.

In this article, we adopt the last definition as also extensively used in real-time scheduling [16, $28,48]$. In this context, it is intuitive to observe that any linear and non-linear increasing function of time with a cutoff at $T_{\text {max }}$ can be used for a suitable penalty function. We choose an exponential function $\phi(t)$, where $t$ is the delivery time, as commonly adopted in soft deadline modeling [3]. It is defined as follows:

$$
\phi(t)= \begin{cases}e^{\alpha\left(t-T_{d}\right)} & \text { if } T_{d}<t \leq T_{\max } \\ \infty & \text { if } t>T_{\max } \\ 0 & \text { otherwise }\end{cases}
$$

Here, $\alpha$ is an arbitrary non-negative value that controls the penalty growth rate.

\subsection{ScE Problem Formulation}

As aforestated, we formulate the ScE path determination problem as a non-linear programming (NLP) optimization problem. The ScE path problem aims at determining, for any message, an optimal path that maximizes energy efficiency, and simultaneously, minimizes the penalty.

Given a directed graph $G=(V, S, E)$, a source node $u \in V$, a destination $v \in V$, a message $m:<$ $u, v, L, T_{d}, T_{\max }>$, and each link $e_{i j}^{(s)} \in E$ characterized by the energy cost $w_{i j}^{(s)}(L)$ and the latency 
cost $\hat{t}_{i j}^{(s)}(L)$, the ScE problem minimizes the following objective function:

$$
\min _{p(u, v) \in P^{\prime}(u, v)}\left(\sum_{e_{i j}^{(s)} \in p(u, v)} w_{i, j}^{(s)}(L)\right)+\phi\left(\sum_{e_{i j}^{(s)} \in p(u, v)} \hat{t}_{i j}^{(s)}(L)\right) .
$$

Here $P^{\prime}(u, v) \subset P(u, v)$ is the set of all paths from the source node $u$ to the destination node $v$ which meets the first four constraints related to the SINR, maximum power, transmission coverage, and unique spectrum described in Section 5.1.

\subsection{ScE Greedy Heuristic}

Following the HcE greedy heuristic, we propose an efficient greedy heuristic to determine the ScE path $p(u, v)$ for any message with soft deadline in the DSA overlay network. As shown in Algorithm 2 (Section 5.3), the HcE greedy heuristic computes the least weighted path $P_{u v}^{\beta}$ from source $u$ to destination $v$ using the values of the weight matrix $H_{i j}^{\beta}$ as edge weights. Then, it computes the total energy cost $E\left(P_{u v}^{\beta}\right)$, latency cost $T\left(P_{u v}^{\beta}\right)$ for least weighted path $P_{u v}^{\beta}$. And, finally the $\operatorname{HcE}$ heuristic chooses the path $p^{*}(u, v)$ as the path with minimum $E\left(P_{u v}^{\beta}\right), \forall \beta$, such that $T\left(P_{u v}^{\beta}\right) \leq T$, the hard deadline. Differently, the ScE heuristic chooses the path $p^{*}(u, v)$ as the path that minimizes the objective function in Equation (6.2) across all values of $\beta$, that is

$$
p^{*}(u, v)=\left\{P_{u v}^{\beta} \mid \min _{\beta} E\left(P_{u v}^{\beta}\right)+\phi\left(T\left(P_{u v}^{\beta}\right)\right)\right\} .
$$

We omit the pseudo code of the algorithm, as it can be easily obtained by replacing line 9 of Algorithm 2 with the above equation. This heuristic will also find a solution as long as one exists according to Theorem 5.2. The time complexity of the ScE greedy heuristic is $O\left(\frac{1}{\Delta \beta}|V|^{2}\right)$, which is similar to that of HcE heuristic.

\section{COST-BENEFIT ANALYSIS AND IMPROVED SUSTAINABILITY}

Many detractors of sustainability argue that green computing incurs higher capital expenditure and operational cost that drains the economy/revenue and makes such designs infeasible in practice. We claim that our proposed approach to dynamic band selection for designing green communication systems, is mostly free from such disadvantages due to following reasons: (i) The use of DSA-enabled access philosophy does not require a provider to own dedicated spectrum licenses, which is worth millions of dollars, and (ii) Multiple smart communities can be connected by a small set of DSA overlay nodes, each costing on the average around $\$ 1700-\$ 1900$ [37]. In contrast, our design obviates the need for new dedicated infrastructure such as macro cellular base station (\$300,000/station) or micro cellular base stations $(\$ 100,000)[24]$ in rural areas and the need for extensive wire line communications to increase the available bandwidth in urban areas. Some real examples of DSA-based deployment has already taken place in relatively rural areas in the developing world, such as Papua [21], Rural South Africa [41], Mexico [17], and the Philippines [23].

To summarize, there is sufficient practical evidence to suggest that the equipment and operational costs of DSA overlay topology are far less compared to the traditional approaches. The major hurdles are the policy issues that surround spectrum planning. Significant breakthroughs in spectrum policy $[35,36]$ have been made in recent years; moreover, DSA-enabled devices mounted over drones, smart public transport [10], and private smart cars [66] are becoming a reality. Let us reiterate that our proposed architecture and solution approach work on top of existing infrastructures (e.g., public buses) to be mounted with a DSA radio device (e.g., ER-N210 [37]) capable 
of dynamic band access. As a result, our approach notably improves energy efficiency of the network via suitable band selection for varying message requirements. Hence, our proposed approach promises to design green yet cost-effective communication systems for realizing smart and connected communities.

The following theorem 7.1 proves that the network energy efficiency can be significantly improved by increasing the number of existing infrastructures, such as public buses or municipal vehicles, mounted with a DSA-enabled device. However, it comes at a higher equipment cost due to the requirement of a larger number of DSA-enabled devices. As part of our future work, we propose to study the tradeoff between the achieved network energy efficiency and equipment cost, for a given rural or urban area.

Theorem 7.1. Given a DSA overlay network $G(V, S, E)$ with a set of messages (say, $M)$, the energy consumed in the $H c E$ (or $S c E)$ path $p(u, v)$ for any message $m \in M$ either improves or remains unaffected due to the introduction of a new DSA node $v^{*}$ in the network.

Proof. The introduction of a new node $v^{*}$ in network $G$, let the modified network become $G^{*}\left(V^{*}, S, E^{*}\right)$ where the node set is $V^{*}=V \cup v^{*}$ and the edge set is given by $E^{*}=E \cup e_{i v^{*}}^{(s)} \quad \forall i \in$ $V, \forall s \in S$, if $d_{i v^{*}}^{(s)} \leq \gamma_{i v^{*}}^{(s)}$. Now, the newly determined HcE (or ScE) path $p^{*}(u, v)$ in the modified $G^{*}$ may (or may not) choose one or more intermediate edges from the set $E^{*} \backslash E$. If the path $p^{*}(u, v)$ chooses at least one intermediate edge from set $E^{\prime} \backslash E$, then the energy consumed in $p^{*}(u, v)$ is less than that of path $p(u, v)$ in the original $G$. If not, then the energy consumed in $p^{*}(u, v)$ in $G^{*}$ is the same as that of $p(u, v)$ in $G$.

Therefore, the energy consumption on the $\mathrm{HcE}$ (or ScE) path $p(u, v)$ either improves or remains unaffected with the introduction of a new DSA node $v^{*}$ in the network $G$. Since the network energy efficiency is the summation of energy savings on each such path for each message $m \in M$, the overall network energy efficiency is also bound to either improve or remain unchanged with increasing number of DSA nodes in the network.

\section{SIMULATION RESULTS}

In this section, we evaluate the performance of the proposed dynamic band selection approach against conventional homogeneous band access approaches for the steady DSA overlay topology under both hard and soft deadlines. The performance metrics include: (i) Energy Efficiency, the average amount of energy consumed to transfer all generated messages from the sensor blocks to their intended data fusion centers; and (ii) Message Delivery Ratio (MDR), the fraction of messages successfully delivered to the fusion center within the delivery deadline (hard or soft) to the total number of messages generated at the sensor blocks.

For the case where messages have hard deadlines over DSA overlay topology, let us first analyze the performance of our proposed approach using optimal DP as well as greedy algorithms for determining HcE paths, against homogeneous band access approaches for determining HcE paths using DP algorithm.

For extensive analysis, we evaluate both the performance metrics considering (i) varying message sizes, (ii) varying hard deadlines, and (iii) varying source-destination node pair distances. The first two parameters represent the message (or QoS) heterogeneity, while the third one represents the geographical heterogeneity. For cost-benefit analysis, we also analyze how the change in the number of DSA nodes in the network (incurring equipment cost) impacts the performance metrics of the proposed band selection approach. Finally, we also provide a detailed analysis on the performance of the proposed dynamic band selection approach under soft deadlines.

Simulation Settings: We simulate a simple SCC scenario with 5 data fusion centers, 15 CPEs, and 30 sensor blocks, each with 50-100 sensing agents. On average, each CPE periodically visits 


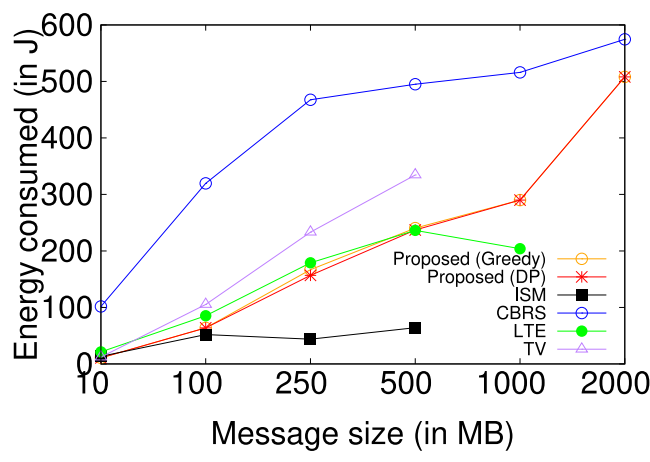

(a)

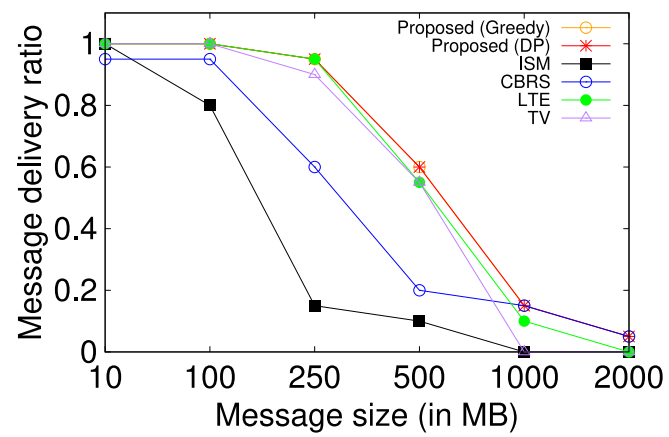

(b)

Fig. 6. Varying message sizes: (a) Energy efficiency and (b) Message Delivery Ratio (MDR).

2 sensor blocks with a round trip time of 1 hour. The steady DSA overlay topology consists of 200 DSA nodes (e.g., public buses, RSUs, etc.). We assume 50\% of overlay nodes (imitating RSUs) are randomly placed and static, whereas other overlay nodes (imitating public buses) move along the prespecified trajectories. We consider that each DSA-enabled node has the knowledge of location and spectrum availability at every other node in the network. Unless otherwise stated, we consider that each sensor block generates messages, each of size $250 \mathrm{MB}$ with hard deadline $T=3$ hours, and the farthest distance between a sensor block and the corresponding fusion center is $d=45 \mathrm{~km}$.

We consider the usually allowable transmit power for all bands on a secondary basis, such as $1 \mathrm{~W}$ for ISM band, $4 \mathrm{~W}$ for TV and LTE bands, and $10 \mathrm{~W}$ for CBRS band. Recall that each band has a FCC mandated maximum allowable transmit power that cannot be exceeded by any node operating on a DSA basis. Finally, realistic values of other controlling parameters are considered as follows: path loss factor $\alpha=3.5$ (sub-urban area), SINR $=-75 \mathrm{dBm}\left(3.16 \times 10^{-8} \mathrm{~mW}\right)$, received power threshold $(\tau)=-20 \mathrm{dBm}(0.01 \mathrm{~mW})$, sensing power $\tilde{P}=40 \mathrm{~mW}$, probability of switching to the idle channel $\mathcal{P}$ is 0.5 , sensing slot duration $t_{s d}=0.1 \mathrm{~s}$, transmission slot duration $t_{t d}=1 \mathrm{~s}$, and energy cost of one channel switching $w_{s w}=1 \mathrm{~mJ}$.

\subsection{Message Heterogeneity}

This section evaluates the performance of the proposed band selection approach against conventional homogeneous band access approaches for varying message sizes and hard deadlines.

8.1.1 Varying Message Sizes:. As shown in Figure 6(a), and (b), the proposed dynamic band selection approach outperforms the homogeneous band access approaches in terms of both energy efficiency and message delivery ratio (MDR). The proposed approach achieves an average of $19 \%$ energy savings compared to the best homogeneous band access approach (i.e., LTE Band), particularly for message size $L \leq 500 \mathrm{MB}$. The reason is evident as the proposed band selection approach utilizes suitable band at each intermediate DSA node so as to attain the most energy-efficient path for any given message. For larger message sizes, $L>500 \mathrm{MB}$, the energy consumption by homogeneous band access approaches (except CBRS band) is missing as none of these bands (i.e., ISM, LTE and TV bands) are able to successfully deliver any message of size $L>500 \mathrm{MB}$ to the fusion center within the hard deadline, i.e., $\mathrm{MDR}=0$. Note that the ISM band consumes significantly smaller energy; however, it suffers from severely poor MDR and hence does not meet the QoS requirements. The greedy heuristic for the proposed approach yields similar energy efficiency as that of the optimal DP algorithm. 


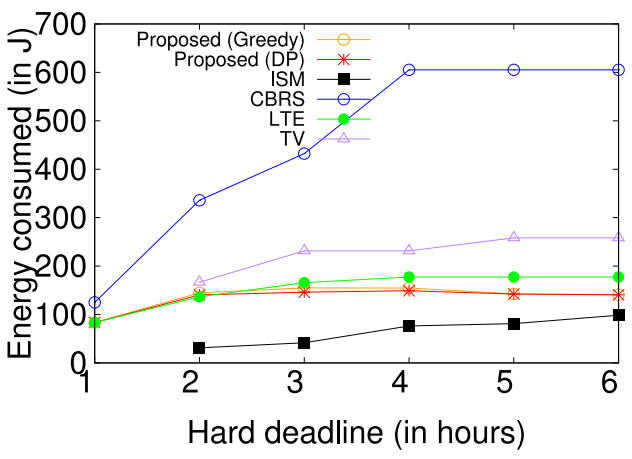

(a)

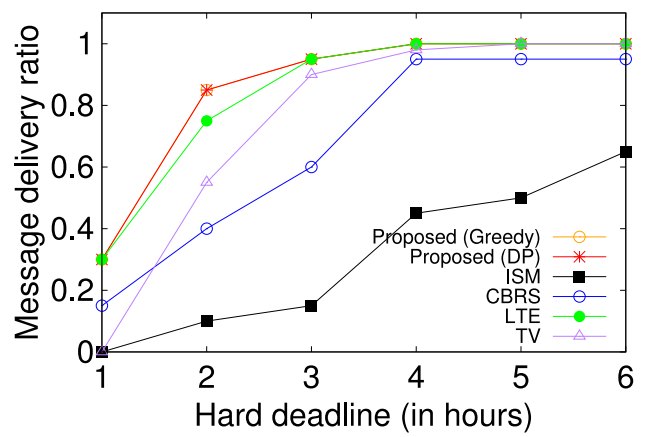

(b)

Fig. 7. Varying hard deadlines: (a) Energy efficiency and (b) MDR.

Our proposed approach yields similar or slightly better MDR (up to 5\%) compared to the best homogeneous approach, i.e., LTE band for message size $L \leq 500 \mathrm{MB}$, and CBRS band for larger message sizes. This is because, unlike homogeneous approaches where the node is restricted to access only one predetermined band, the proposed approach intelligently chooses the most suitable energy-efficient band at each intermediate node while guaranteeing the hard deadline for every message and hence MDR or QoS. Recall that for any given message, both the DP algorithm (owing to being optimal) and greedy algorithm (from Theorem 5.2) are able to determine a HcE path (if it exists) for a given source-destination pair that meets the hard deadline, and hence always guarantee the highest MDR.

8.1.2 Varying Hard Deadlines:. Figure 7(a) shows that the proposed band selection approach yields better energy efficiency compared to that of the homogeneous band access approaches for increased hard deadlines. Our approach saves energy consumption by up to $22 \%$ on the average compared to the best homogeneous band (i.e., LTE band) provided that it also guarantees comparable MDR. Here again, the ISM band yields the best energy efficiency, thanks to the low transmit power; however, it suffers from poor MDR (refer to Figure 7(b)). The CBRS band yields very poor energy efficiency, because the transmit power is very high compared to its counterparts.

As shown in Figure 7(b), the proposed band selection approach achieves similar MDR compared to the homogeneous band access approaches. This is because our proposed approach for HcE path determination simultaneously accounts for hard delivery deadlines besides enhancing energy savings. Since the homogeneous band approaches do not have the flexibility of choosing any other band than the prespecified one, they perform relatively poorly. Note that both the greedy heuristic and the DP algorithm work almost equally well in improving energy efficiency and MDR irrespective of varying hard delivery deadlines.

\subsection{Geographical Heterogeneity}

As depicted in Figure 8(a) and (b), the dynamic band selection approach outperforms the homogeneous band access approaches in terms of both energy efficiency and MDR for varying sourcedestination node-pair distances (this is geographical heterogeneity). The proposed approach enhances energy efficiency by up to $32 \%$ compared to the best homogeneous band access approach that also yields comparable MDR, particularly for distance $d \leq 60 \mathrm{~km}$. We observe that the CBRS band yields very poor energy efficiency until distance $d \leq 45 \mathrm{~km}$ and then improves significantly. This is because the CBRS band, owing to its limited transmission coverage, is not able to deliver messages successfully, and therefore do not dissipate energy in message transmission, for larger 


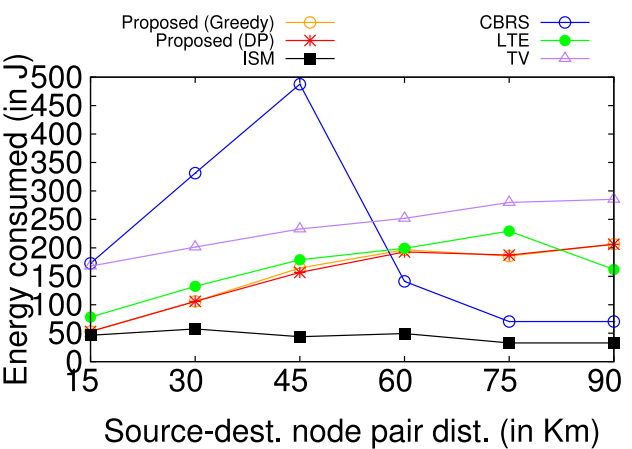

(a)

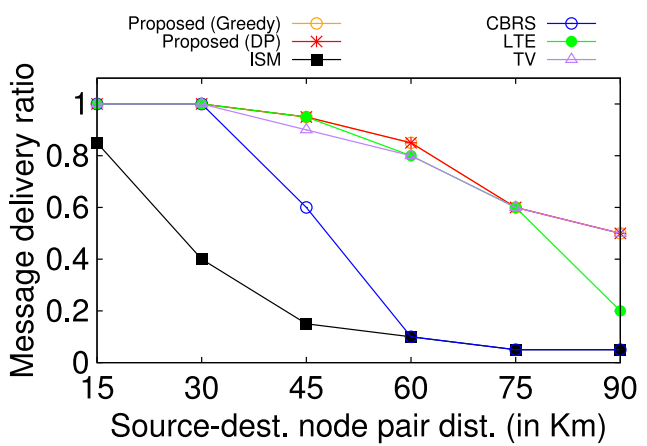

(b)

Fig. 8. Geographical heterogeneity: (a) Energy efficiency and (b) MDR.

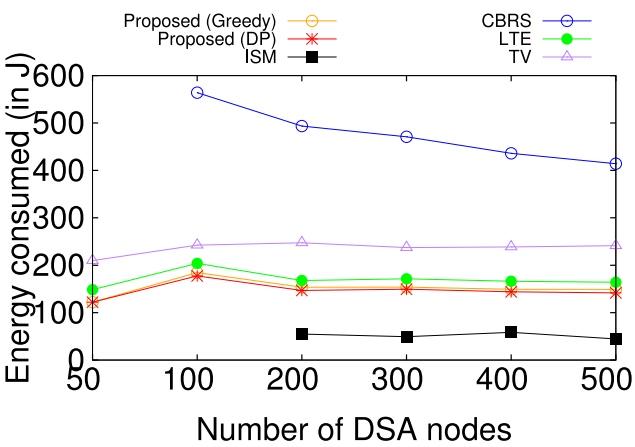

(a)

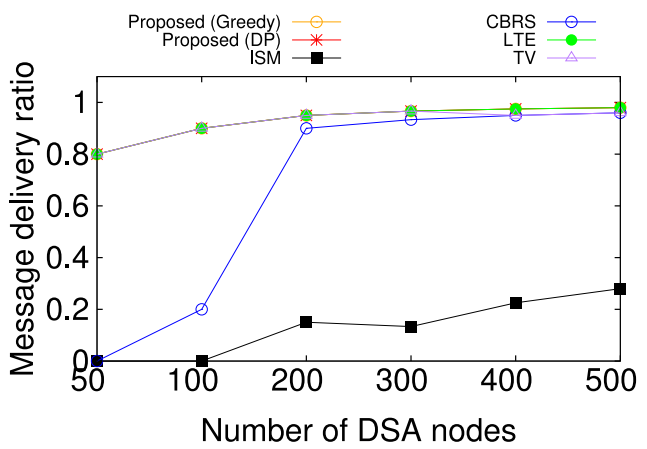

(b)

Fig. 9. Varying number of DSA nodes: (a) Energy efficiency and (b) MDR.

distances between source-destination node-pairs. The ISM band again owing to its low transmit power, yields significantly better energy efficiency; however it suffers greatly in terms of MDR, thus defeating the purpose of SCCs.

Our proposed approach achieves slightly better MDR (about 7\% on an average) compared to the LTE Band, the best homogeneous band selection approach. The reasons are as explained above. The greedy heuristic works very well in determining optimal HcE path and hence yields similar energy efficiency and MDR as that of the DP algorithm employed for band selection.

\subsection{Cost-Benefit Analysis}

We evaluate the benefits of our band selection approach in terms of both energy efficiency and MDR (QoS) against varying number of DSA nodes in the overlay network (which is equivalent to the incurred equipment cost).

As shown in Figure 9(a) and (b), the proposed band selection approach largely outperforms all homogeneous band access approaches in terms of both energy efficiency and MDR, irrespective of the number of DSA nodes. Our approach enhances energy efficiency by up to $18 \%$ compared to the best homogeneous band (i.e., LTE Band). Furthermore, the energy consumption increases with increase in the number of DSA nodes until $|V|=100$. After that, it gradually decreases until $|V|=$ 300 nodes and remains almost unchanged afterwards. This is because initially $|V|$ itself is small and yields slightly lower MDR, thus the energy consumption in delivering messages is also less. 


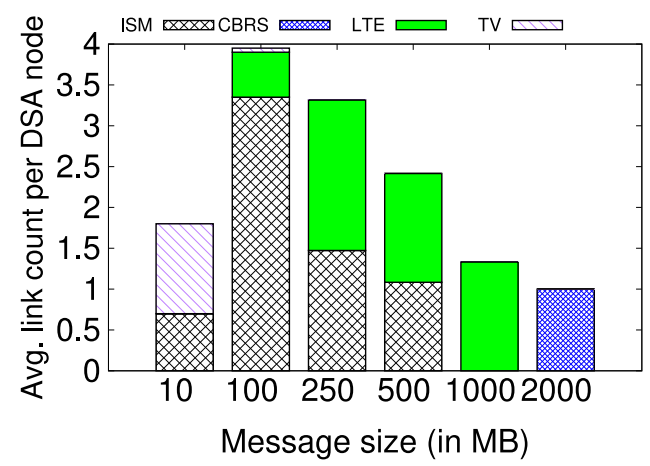

(a)

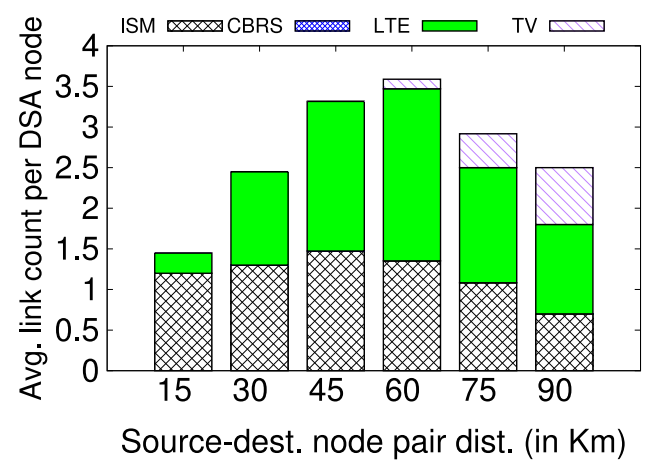

(c)

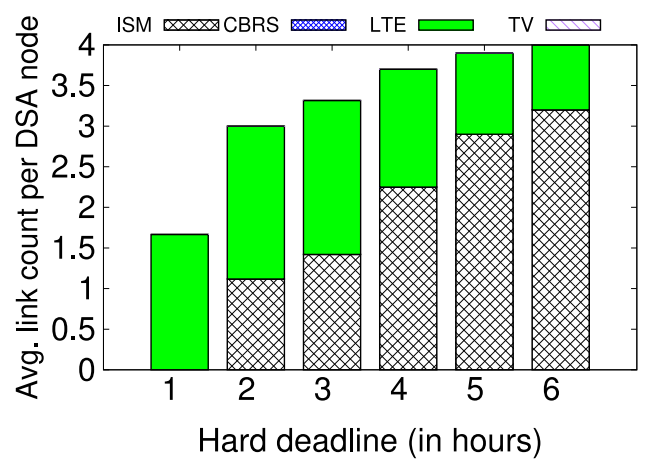

(b)

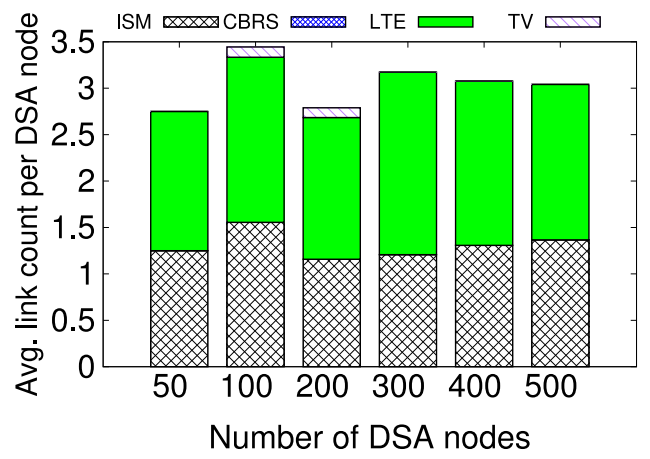

(d)

Fig. 10. Band selection analysis: (a) message sizes, (b) hard deadlines, (c) source-destination node pair distances, and (d) number of DSA nodes.

In contrast, when $|V|$ is sufficiently large, say, $\geq 200$, the proposed approach is able to determine better energy-efficient paths and so consumes less energy at each intermediate node (as shown in Theorem 7.1) while achieving very high MDR.

Energy consumed by ISM and CBRS bands are $0 \mathrm{~J}$ for $|V| \leq 200$ and 100 , respectively. This is because none of the messages was delivered to their intended fusion centers within hard deadlines; therefore they are not desirable for data communication in SCCs (see Figure 9(b)). Moreover, the greedy heuristic also determines near-optimal HcE paths and achieves similar energy efficiency and MDR as that of the DP algorithm using the proposed approach.

\subsection{Band Selection Analysis}

This section investigates how our proposed band selection approach dynamically chooses suitable band types at the intermediate DSA nodes for each message, thereby enhancing energy efficiency of the network while meeting the QoS. As shown in Figure 10(a)-(d), the proposed approach extensively utilizes the ISM and LTE bands, compared to the other two remaining bands. This is reasonable given that the simulated SCC scenario is characterized by $|V|=200$ and $d=45 \mathrm{~km}$, forming a relatively dense DSA network topology. Now, given that the message size $L=250 \mathrm{MB}$ and the hard deadline $T=3$ hours, it becomes a suitable case for ISM and LTE bands to achieve energy-efficient data communications. This is because these bands are characterized with relatively low transmit power, good bandwidth and comparatively good coverage. 
However, as shown in Figure 10(a), for message size $L<250 \mathrm{MB}$, our dynamic band selection approach also utilizes the TV band for energy-efficient message transmissions. This is because the TV band offers energy-efficient HcE paths for lower message sizes, thanks to its sufficiently adequate bandwidth $(6 \mathrm{MHz})$ and very high transmission coverage (several $\mathrm{kms})$. It is interesting to note that messages with size $L=2000 \mathrm{MB}$ were delivered with hard deadline over the CBRS band only. The reasons is unlike other bands, the CBRS band offers very high bandwidth and therefore requires very low transmission time and meets hard deadlines for larger message sizes.

As shown in Figure 10(b), the proposed approach utilizes the ISM band more with increasing hard deadline. This is evident because this band, owing to its low transmit power, can now be utilized along energy-efficient paths that meet hard deadlines, for larger number of messages.

Similarly, as shown in Figure 10(c), for larger source-destination node-pair distances $d>45 \mathrm{~km}$, the proposed approach again significantly utilizes the TV band for delivering messages in an energy-efficient manner. Intuitively, the TV bands offer high transmission coverage, and thus proves to be useful bands for energy-efficient communications for higher distances. Note that the CBRS bands are rarely utilized by the proposed approach due to the fact that it offers limited coverage and requires very high transmit power.

Finally, as shown in Figure 10(d), the proposed approach utilizes the ISM band more for increasing number of DSA nodes. This implies, as the network gets denser, the ISM band can be utilized for energy-efficient communications at the intermediate nodes of $\mathrm{HcE}$ paths for larger number of messages.

\subsection{Soft Deadlines Analysis}

In this section, we study the performance of the proposed band selection approach for the steady DSA overlay topology where messages have soft deadlines. For the experiments, we consider the same SCC scenario discussed in the simulation settings. The values of all parameters including the message size, the number of DSA nodes, and source-destination distance are also kept unchanged. Finally, we set the desired deadline to $T_{d}=2$ hours. The performances are discussed in terms of message delivery ratio (MDR) and energy efficiency against the maximum tardiness $T_{\max }$ and the penalty growth rate $\alpha$ defining the penalty function (see Equation (15)).

Figure 11(a) shows the values of the penalty function under several settings of $\alpha$ and $T_{\text {max }}$. As expected, the penalty value is 0 before the desired deadline $T_{d}$ (2 hours in our experiments), irrespective of the growth rate, $\alpha$. However, after $T_{d}$, the penalty value grows exponentially, and different values of $\alpha$ strongly influence the growth rate, making the penalty value more or less dominant in the objective function in Equation (6.2). This aspect will be necessary to understand the following results.

Figure 11(b) demonstrates the energy consumption by increasing $T_{\max }$, under different settings of $\alpha$. By keeping the desired deadline $T_{d}$ fixed and increasing $T_{\max }$, there are more potential paths that can be followed. As a result, we can initially deliver more messages, and consequently increase the energy consumption until the MDR saturates around 4 hours (see Figure 11(c)). After that time, the value of $\alpha$ determines the trend of the energy consumption. Specifically, if $\alpha$ is low $(0.01,0.05)$, the impact of the penalty function is minimal, and the algorithm would target paths that may deliver the messages later but are more energy efficient. Conversely, if $\alpha$ is high (say, $\geq 0.1$, the penalty function makes these paths unattractive, orienting the algorithm towards less energy-efficient paths that would deliver the messages sooner. To further support this observation, Figure 11(d) shows the breakdown of the average link counts per DSA nodes for varying values of penalty growth rate $\alpha$. Clearly, as the penalty function becomes more dominant, the LTE bands are preferred over the ISM bands, since they lower transmission times at the cost of higher energy consumption, as discussed in Section 4. 


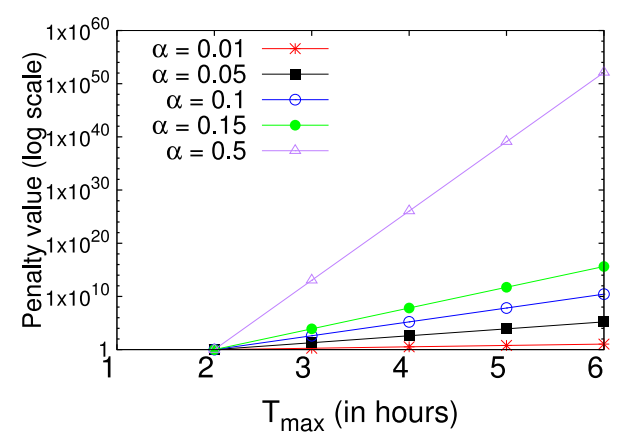

(a)

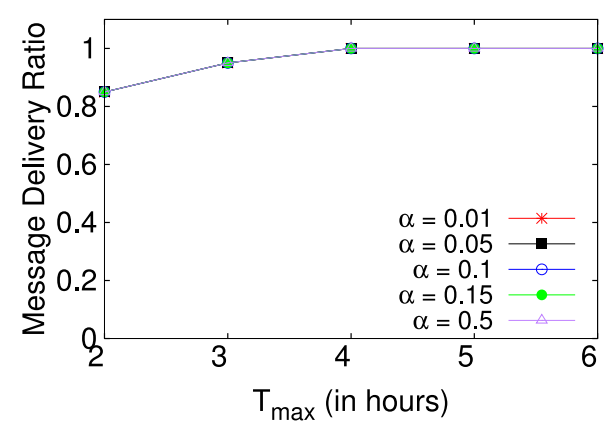

(c)

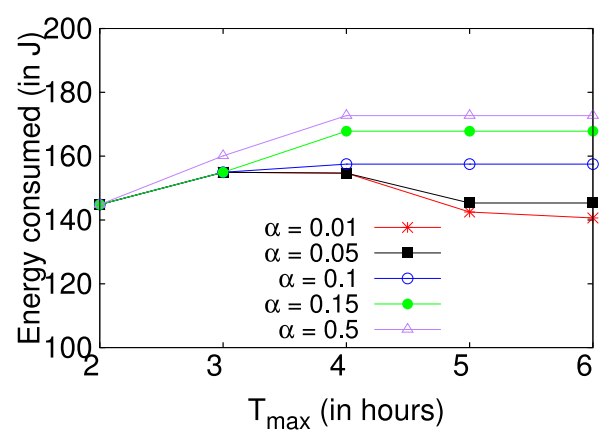

(b)

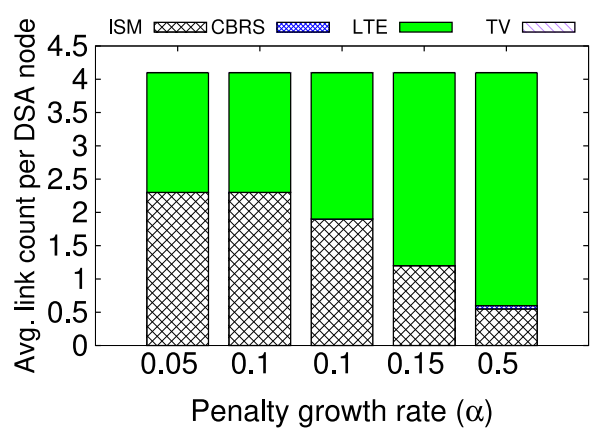

(d)

Fig. 11. Soft deadlines: (a) energy efficiency, (b) Message Delivery Ratio (MDR), and (c) band selection.

Figure 11(c) depicts the MDR for varying $T_{\max }$ under different settings of $\alpha$. Intuitively, MDR is bound to increase with the increase in $T_{\text {max }}$. However, the MDR remains unaffected by change in $\alpha$. This is because, provided there exists at least one valid path for any message that meets soft deadline requirement, our proposed heuristic would always find a solution (similar to Theorem 5.2 for messages with hard deadlines).

We do not show the plots for homogeneous band selection approach as they remain unaffected by the change in $T_{\max }$ or $\alpha$. This is because, as shown in Equations (2) and (3), the message transmission time is directly proportional to the energy consumed for a fixed pre-determined band. Hence determining an energy-efficient path is equivalent to determining a path with the least transmission time. It is intuitive that energy efficiency and MDR achieved by our proposed approach would be significantly better than the homogeneous band access approaches, irrespective of varying $T_{\max }$ values and lower $\alpha$ values. Even for a higher value of $\alpha$, the energy efficiency achieved by our proposed approach is either better or comparable to the best homogeneous (LTE) band as shown in Figure 11(d). Finally, we observe that the MDR and energy efficiency results corresponding to the soft deadlines are consistent with those corresponding to the hard deadlines for varying contextual factors, such as message sizes, source-destination node pair distances, and the number of DSA nodes. Such plots are not shown here for brevity.

To summarize, the proposed band selection approach outperforms the homogeneous band access approaches for any message with hard or soft deadlines, in terms of both energy efficiency and MDR under all contextual factors such as message and geographical heterogeneities. This concludes that the dynamic band selection is a promising approach for designing energy-efficient and high quality communication systems for sustainable SCCs in both rural and urban areas. 


\section{VARIABLE DSA TOPOLOGY: A CASE STUDY AND FUTURE WORK}

To realize the true power of ubiquitous connectivity in SCCs, we believe private smart vehicles of the future could also be harnessed as integral building blocks of the DSA overlay network. However, since mobility in such cases is not predictable due to variable routes, constructing a global knowledge may be infeasible. We explore certain challenges and a road map for energyefficient band selection under such topologies. Due to the lack of global knowledge, we investigate a simple greedy approach that attempts to choose the locally optimized energy-efficient band at any given node for any message $m\langle u, v, L, T\rangle$, aiming to provide end-to-end energy efficiency while meeting a prespecified hard delivery deadline. A similar approach can be adopted to consider soft deadlines by simply changing the local optimized metric and including the penalty function.

This greedy algorithm chooses the band $s \in S$ at any given node $u$ for a message $m$ only if the following two conditions are met: (i) If the next hop is the destination node and (ii) the energy consumed over band $s$ is the least, then the latency cost is less than the deadline, and there exists at least two nodes in the communication range over band $s$. These conditions are important to improve the chances of successful data delivery to the destination node. This is formally presented in Algorithm 3. Once the suitable band is chosen at the current node, it sends a message copy over the chosen band $s$ to all the neighboring nodes that lie in the communication range. The time complexity of the algorithm is $O(|V| \times|S|)$ where $V$ is the set of nodes and $S$ is the set of bands.

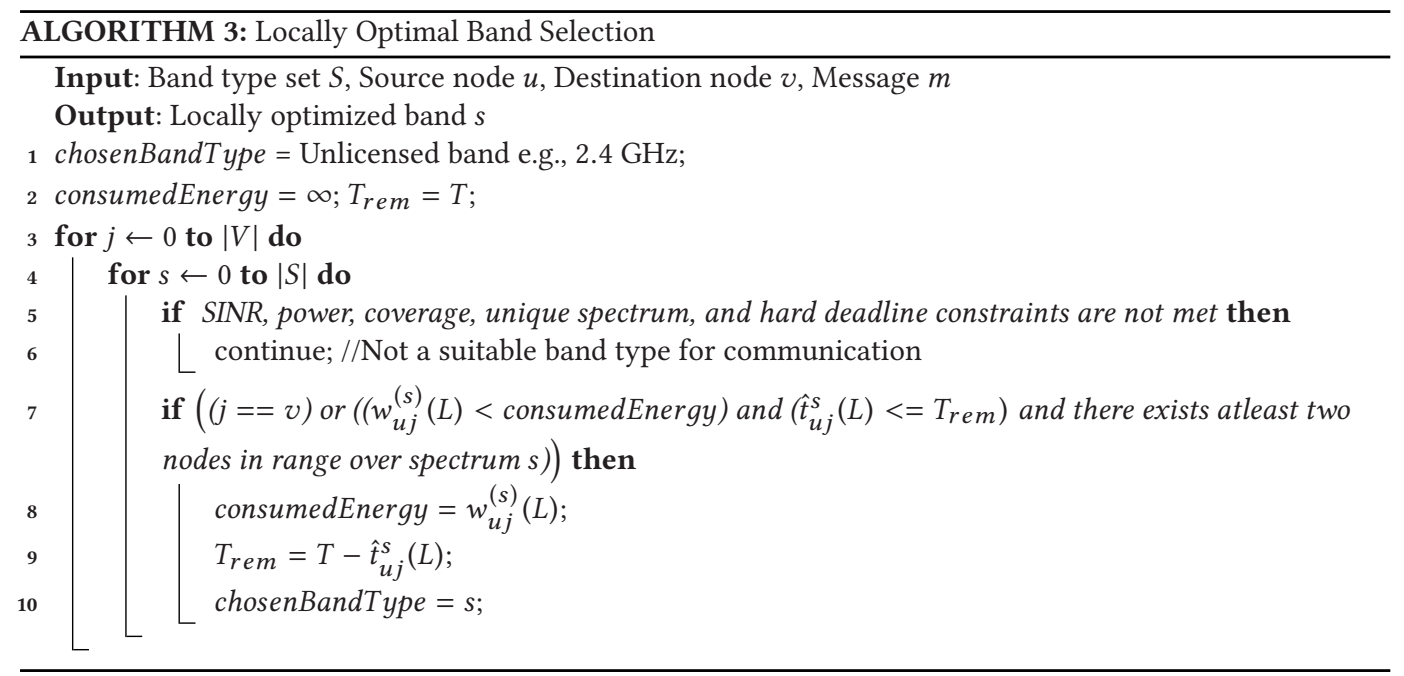

Challenges and Future Roadmap. Now we investigate the performance of the greedy local optimization in variable DSA topology, and draw key challenges and a future roadmap for further research. For the performance analysis, we consider the similar simulation setting as presented in Section 8. However, in this case, each DSA node moves randomly in the area. Furthermore, each DSA-enabled node only has the knowledge of location and spectrum availability at its neighboring nodes.

From Figure 12(b), it is evident that although the greedy approach outperforms others in terms of MDR, it suffers greatly in terms of energy efficiency compared to other homogeneous band access approaches for varying message sizes (see Figure 12(a)). This greedy approach also chooses the LTE band majority of the times (see Figure 12(c)) for data communication similar to that of the steady DSA topology. However, the greedy approach yields poor energy efficiency mainly due to 


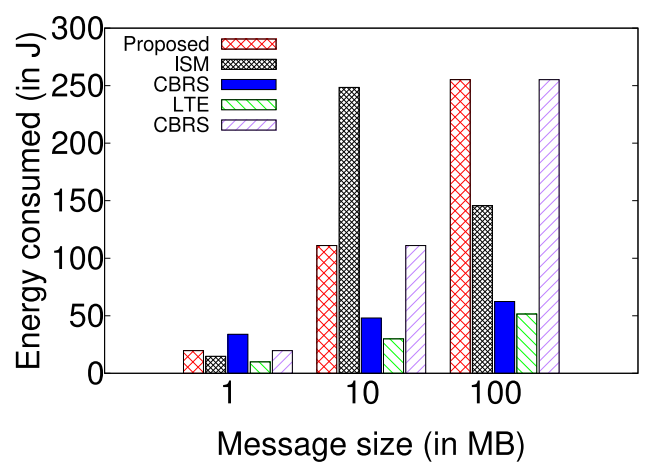

(a)

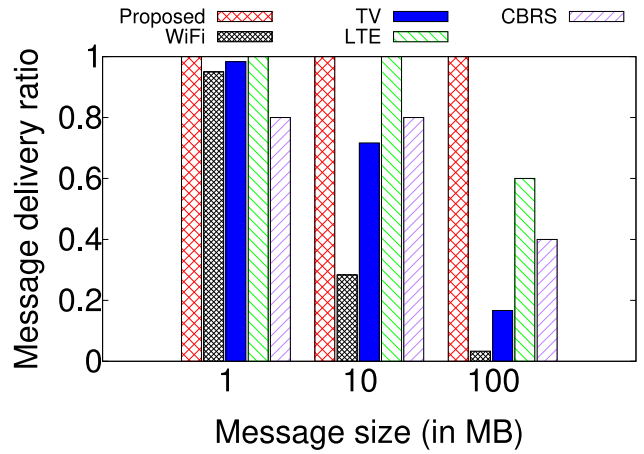

(b)

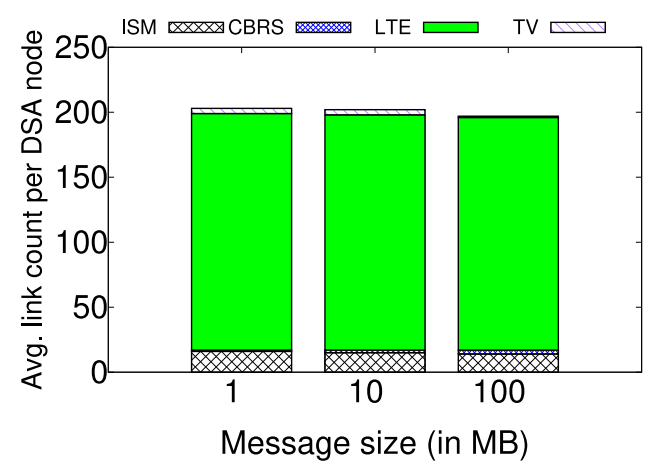

(c)

Fig. 12. Message sizes: (a) energy efficiency, (b) MDR, and (c) band selection.

contextual factors, such as unpredictable node mobility patterns, node contact behaviors, and so on. The results being similar to other two parameters, those plots are not shown here.

We conclude that a locally optimized greedy solution alone is not an apt solution to improve end-to-end energy efficiency via dynamic band selection. In fact, the optimization under that case should be complemented by an efficient routing protocol that reduces the unpredictability of determining the best set of intermediate nodes by learning geographical contextual factors and contact mobility patterns. As part of future work, we plan to investigate these contextual factors and come up with a spectrum and mobility aware approach that improves end-to-end energy efficiency for any given message yet satisfying hard (and soft) deadlines for a variable DSA topology.

\section{CONCLUSIONS}

In this article, we proposed a novel architecture that uses a small scale DSA overlay network on top of the legacy infrastructure, for designing green and high quality communication systems to provide ubiquitous connectivity in SCCs, be it rural or urban. We discussed how the proposed dynamic band selection approach intelligently matches any message requirement at each DSA-enabled node, to a suitable band type for energy-efficient data communication by exploiting electro-magnetic characteristics of various bands. Additionally, we formulated the determination of hard deadline constrained energy-efficient $(\mathrm{HcE})$ path as a linear optimization problem and proved that it is NP-Hard. Subsequently, we proposed a pseudo-polynomial dynamic programming 
algorithm to solve the HcE problem exactly and determine the optimal HcE path (and hence optimal band at each intermediate node) for any given message. We further proposed a greedy heuristic that provides a faster yet effective solution to the HcE problem. Additionally, we investigated the dynamic band selection approach under scenarios where messages have soft deadlines. Compared to the homogeneous band access approaches, our proposed dynamic approach significantly improved the network energy efficiency while meeting various hard and soft delivery deadlines, irrespective of the message and geographical heterogeneities. Thus our approach bypasses underprovisioning and over-provisioning issues, policy constraints, and the requirement of a dedicated infrastructure. Finally, we investigated and discussed the challenges of optimal band selection for a variable DSA topology as a case study, which we intend to explore further in future.

\section{ACKNOWLEDGMENTS}

The authors thank the anonymous reviewers and the Associate Editor for their detailed and constructive feedback that greatly strengthened the contribution and quality of this article. The authors also thank Rishabh Singhal, an undergraduate intern, for his assistance with the simulation experiments.

\section{REFERENCES}

[1] Satyam Agarwal and Swades De. 2016. eDSA: Energy-efficient dynamic spectrum access protocols for cognitive radio networks. IEEE Trans. Mob. Comput. 15, 12 (2016), 3057-3071.

[2] Ian F. Akyildiz, Won-Yeol Lee, Mehmet C. Vuran, and Shantidev Mohanty. 2006. NeXt generation/dynamic spectrum access/cognitive radio wireless networks: A survey. Comput. Netw. 50, 13 (2006), 2127-2159.

[3] M. D. Nashid Anjum and Honggang Wang. 2016. Dynamic scheduling and analysis of real time systems with multiprocessors. Dig. Commun. Netw. 2, 3 (2016), 130-138.

[4] M. Aubree, D. Marquet, S. Le Masson, H. Louahlia, A. Chehade, J. David, and F. Van Goethem. 2014. OPERA-Net 2 project: An environmental global approach for radio access networks-achievements for off-grid systems. In Proceedings of the 2014 IEEE 36th International Telecommunications Energy Conference (INTELEC'14). IEEE, 1-7.

[5] Junjik Bae, Eyal Beigman, Randall Berry, Michael L Honig, Hongxia Shen, Rakesh Vohra, and Hang Zhou. 2008. Spectrum markets for wireless services. In Proceedings of the 3rd IEEE Symposium on New Frontiers in Dynamic Spectrum Access Networks, 2008 (DySPAN'08). IEEE, 1-10.

[6] Pietro Belotti, Christian Kirches, Sven Leyffer, Jeff Linderoth, James Luedtke, and Ashutosh Mahajan. 2013. Mixedinteger nonlinear optimization. Acta Numer. 22 (2013), 1-131.

[7] Yahel Ben-David, Matthias Vallentin, Seth Fowler, and Eric Brewer. 2010. JaldiMAC: Taking the distance further. In Proceedings of the 4th ACM Workshop on Networked Systems for Developing Regions. ACM, 2.

[8] Flavio Bonomi, Rodolfo Milito, Preethi Natarajan, and Jiang Zhu. 2014. Fog computing: A platform for internet of things and analytics. In Big Data and Internet of Things: A Roadmap for Smart Environments. Springer, 169-186.

[9] Swastik Brahma and Mainak Chatterjee. 2012. Spectrum sharing in secondary networks: A bargain theoretic approach. In Wireless Communications and Networking Conference (WCNC), 2012 IEEE. IEEE, 1331-1336.

[10] Lin Cheng, Benjamin E. Henty, Daniel D. Stancil, Fan Bai, and Priyantha Mudalige. 2007. Mobile vehicle-to-vehicle narrow-band channel measurement and characterization of the $5.9 \mathrm{GHz}$ dedicated short range communication (DSRC) frequency band. IEEE 7. Select. Areas Commun. 25, 8 (2007).

[11] Kaushik R. Chowdhury and Ian F. Akyildiz. 2011. CRP: A routing protocol for cognitive radio ad hoc networks. IEEE f. Select. Areas Commun. 29, 4 (2011), 794-804.

[12] Michael Corinthios. 2009. Signals, Systems, Transforms, and Digital Signal Processing with MATLAB. CRC Press.

[13] Thomas H. Cormen, Charles E. Leiserson, Ronald L. Rivest, and Clifford Stein. 1990. Introduction to Algorithms. MIT Press, Cambridge MA.

[14] Saptarshi Debroy, Shameek Bhattacharjee, and Mainak Chatterjee. 2015. Spectrum map and its application in resource management in cognitive radio networks. IEEE Trans. Cogn. Commun. Netw. 1, 4 (2015), 406-419.

[15] Saptarshi Debroy, Swades De, and Mainak Chatterjee. 2014. Contention based multichannel MAC protocol for distributed cognitive radio networks. IEEE Trans. Mobile Comput. 13, 12 (2014), 2749-2762.

[16] Umamaheswari C. Devi and James H. Anderson. 2006. Soft Real-time Scheduling on Multiprocessors. University of North Carolina at Chapel Hill.

[17] Hernan Galperin and François Bar. 2006. The microtelco opportunity: Evidence from Latin America. Inf. Technol. Int. Dev. 3, 2 (2006), 73. 
[18] Mohammad Ghamari, Balazs Janko, R. Simon Sherratt, William Harwin, Robert Piechockic, and Cinna Soltanpur. 2016. A survey on wireless body area networks for ehealthcare systems in residential environments. Sensors 16, 6 (2016), 831.

[19] P. Grant. 2010. MCVE Core 5 Programme, Green radio-the case for more efficient cellular basestations. Globecom.

[20] Markus Gruber, Oliver Blume, Dieter Ferling, Dietrich Zeller, Muhammad Ali Imran, and Emilio Calvanese Strinati. 2009. EARTH: Energy aware radio and network technologies. In Proceedings of the 2009 IEEE 20th International Symposium on Personal, Indoor and Mobile Radio Communications. IEEE, 1-5.

[21] Shaddi Hasan, Kurtis Heimerl, Kate Harrison, Kashif Ali, Sean Roberts, Anant Sahai, and Eric Brewer. 2014. GSM whitespaces: An opportunity for rural cellular service. In Proceedings of the 2014 IEEE International Symposium on Dynamic Spectrum Access Networks (DYSPAN'14). IEEE, 271-282.

[22] Kurtis Heimerl and Eric Brewer. 2010. The village base station. In Proceedings of the 4th ACM Workshop on Networked Systems for Developing Regions. ACM, 14.

[23] Kurtis Heimerl, Shaddi Hasan, Kashif Ali, Eric Brewer, and Tapan Parikh. 2013. Local, sustainable, small-scale cellular networks. In Proceedings of the 6th International Conference on Information and Communication Technologies and Development: Full Papers-Volume 1. ACM, 2-12.

[24] Klas Johansson, Anders Furuskar, Peter Karlsson, and Jens Zander. 2004. Relation between base station characteristics and cost structure in cellular systems. In Proceedings of the 15th IEEE International Symposium on Personal, Indoor and Mobile Radio Communications, 2004 (PIMRC'04), Vol. 4. IEEE, 2627-2631.

[25] Mohsin Khalil, Junaid Qadir, Oluwakayode Onireti, Muhammad Ali Imran, and Shahzad Younis. 2017. Feasibility, architecture and cost considerations of using TVWS for rural internet access in 5G. In Proceedings of the 201720 th Conference on Innovations in Clouds, Internet and Networks (ICIN'17). IEEE, 23-30.

[26] Yara Khaluf and Franz J Rammig. 2013. Task allocation strategy for time-constrained tasks in robot swarms. In Proceedings of the European Conference on Artificial Life (ECAL'13). 737-744.

[27] Turgay Korkmaz and Marwan Krunz. 2001. Multi-constrained optimal path selection. In Proceedings of the 20th Annual foint Conference of the IEEE Computer and Communications Societies (INFOCOM'01), Vol. 2. IEEE, 834-843.

[28] Hennadiy Leontyev and James H. Anderson. 2008. A unified hard/soft real-time schedulability test for global EDF multiprocessor scheduling. In Proceedings of the Real-Time Systems Symposium, 2008. IEEE, 375-384.

[29] Ding Li and William G. J. Halfond. 2014. An investigation into energy-saving programming practices for android smartphone app development. In Proceedings of the 3rd International Workshop on Green and Sustainable Software. ACM, 46-53.

[30] Ying-Chang Liang, Anh Tuan Hoang, and Hsiao-Hwa Chen. 2008. Cognitive radio on TV bands: A new approach to provide wireless connectivity for rural areas. IEEE Wireless Commun. 15, 3 (2008).

[31] Yuen T. Lo and S. W. Lee. 2013. Antenna Handbook: Theory, Applications, and Design. Springer Science \& Business Media.

[32] Hrushikesh Mehendale, Ashwin Paranjpe, and Santosh Vempala. 2011. Lifenet: A Flexible Ad Hoc Networking Solution for Transient Environments. Vol. 41. ACM.

[33] Ramyar Rashed Mohassel, Alan Fung, Farah Mohammadi, and Kaamran Raahemifar. 2014. A survey on advanced metering infrastructure. Int. F. Electr. Power Energy Syst. 63 (2014), 473-484.

[34] 2014. Retrieved June 18, 2017 from http://www.rtl-sdr.com/rtl-sdr-wide-spectrum-analyzer/.

[35] Federal Communications Commission and others. 2015. Report and order and second further notice of proposed rulemaking. Amendment of the Commission's Rules with Regard to Commercial Operations in the 3550-3650 MHz Band. $3550-3650$.

[36] FCC, ET. 2010. Docket No. 10-174. Second Memorandum Opinion and Order.

[37] 2015. Retrieved January 12, 2018 https://www.ettus.com/product/details/UN210-KIT.

[38] Internet.org. 2015. Facebook Inc. Retrieved May 14, 2018 from https://www.internet.org/projects.

[39] Project loon. 2015. Retrieved June 14, 2017 from www.google.com/loon.

[40] [Online] GSA Global mobile Suppliers Association. 2016. Retrieved June 18, 2017 from https://gsacom.com/ $5 \mathrm{~g}$-spectrum-bands/.

[41] [Online] Microsoft Limpopo TV White Spaces Project. 2016. Retrieved June 18, 2017 from https://www.microsoft. com/en-us/research/wp-content/uploads/2016/02/spectrum-microsoft_tvwsimpact_limpopo_rural_south_africa. pdf.

[42] [Online] 5G Americas White Paper on 5G Spectrum Recommendations. 2017. Retrieved January 1, 2018 from http: //www.5gamericas.org/files/9114/9324/1786/5GA_5G_Spectrum_Recommendations_2017_FINAL.pdf.

[43] [Online] Energy-efficient WIreless Networking (eWIN). 2018. Retrieved May 30, 2018 from https://www.kth.se/cos/ research/kth-serst-research/current-research-pro/energy-efficient-wireless-networking-ewin-1.311831.

[44] [Online] NSF's Smart \& Connected Communities Effort. 2018. Retrieved on May 30, 2018 from https://www.nsf.gov/ cise/scc/. 
[45] Alexandra-Gwyn Paetz, Elisabeth Dütschke, and Wolf Fichtner. 2012. Smart homes as a means to sustainable energy consumption: A study of consumer perceptions. f. Consum. Pol. 35, 1 (2012), 23-41.

[46] Sushovan Patra, Sujoy Saha, Vijay Shah, Satadal Sengupta, Konsam Gojendra Singh, and Subrata Nandi. 2011. A qualitative survey on multicast routing in delay tolerant networks. In Recent Trends in Wireless and Mobile Networks. Springer, 197-206.

[47] Alex Pentland, Richard Fletcher, and Amir Hasson. 2004. Daknet: Rethinking connectivity in developing nations. Computer 37, 1 (2004), 78-83.

[48] Linh T. X. Phan, Zhuoyao Zhang, Boon Thau Loo, and Insup Lee. 2010. Real-time MapReduce scheduling. (2010).

[49] Asfandyar Qureshi, Rick Weber, Hari Balakrishnan, John Guttag, and Bruce Maggs. 2009. Cutting the electric bill for internet-scale systems. In ACM SIGCOMM Computer Communication Review, Vol. 39. ACM, 123-134.

[50] G. N. Rameshaiah, J. Pallavi, and S. Shabnam. 2015. Nano fertilizers and nano sensors-an attempt for developing smart agriculture. Int. F. Eng. Res. Gen. Sci. 3, 1 (2015), 2091-2730.

[51] Ju Ren, Yaoxue Zhang, Ning Zhang, Deyu Zhang, and Xuemin Shen. 2016. Dynamic channel access to improve energy efficiency in cognitive radio sensor networks. IEEE Trans. Wireless Commun. 15, 5 (2016), 3143-3156.

[52] Sujoy Saha, Subrata Nandi, Partha Sarathi Paul, Vijay K Shah, Akash Roy, and Sajal K Das. 2015. Designing delay constrained hybrid ad hoc network infrastructure for post-disaster communication. Ad Hoc Netw. 25 (2015), 406-429.

[53] Sujoy Saha, Vijay Kumar Shah, Rohit Verma, Ratna Mandal, and Subrata Nandi. 2012. Is it worth taking a planned approach to design ad hoc infrastructure for post disaster communication?. In Proceedings of the 7th ACM International Workshop on Challenged Networks. ACM, 87-90.

[54] Aaditeshwar Seth, Darcy Kroeker, Matei Zaharia, Shimin Guo, and Srinivasan Keshav. 2006. Low-cost communication for rural internet kiosks using mechanical backhaul. In Proceedings of the 12th Annual International Conference on Mobile Computing and Networking. ACM, 334-345.

[55] Vijay K. Shah, Shameek Bhattacharjee, Simone Silvestri, and Sajal K. Das. 2017. Designing sustainable smart connected communities using dynamic spectrum access via band selection. In Proceedings of the 4th ACM International Conference on Systems for Energy-Efficient Built Environments. ACM, 12.

[56] Claude Elwood Shannon. 2001. A mathematical theory of communication. ACM SIGMOBILE Mobile Comput. Commun. Rev. 5, 1 (2001), 3-55.

[57] Hossein Shokri-Ghadikolaei, Ioannis Glaropoulos, Viktoria Fodor, Carlo Fischione, and Antony Ephremides. 2015. Green sensing and access: Energy-throughput trade-offs in cognitive networking. IEEE Commun. Mag. 53, 11 (2015), 199-207.

[58] Kamal D. Singh, Priyanka Rawat, and Jean-Marie Bonnin. 2014. Cognitive radio for vehicular ad hoc networks (CRVANETs): Approaches and challenges. EURASIP f. Wireless Commun. Netw. 2014, 1 (2014), 49.

[59] Min Song, Chunsheng Xin, Yanxiao Zhao, and Xiuzhen Cheng. 2012. Dynamic spectrum access: From cognitive radio to network radio. IEEE Wireless Commun. 19, 1 (2012).

[60] Purnima Surampudi and Sony Mohanty. 2011. LTE-advanced in white space: A complementary technology. Radisys White Paper (2011).

[61] Song Tan, Debraj De, Wen-Zhan Song, Junjie Yang, and Sajal K. Das. 2017. Survey of security advances in smart grid: A data driven approach. IEEE Commun. Surv. Tutor. 19, 1 (2017), 397-422.

[62] Roya H. Tehrani, Seiamak Vahid, Dionysia Triantafyllopoulou, Haeyoung Lee, and Klaus Moessner. 2016. Licensed spectrum sharing schemes for mobile operators: A survey and outlook. IEEE Commun. Surv. Tutor. 18, 4 (2016), 25912623.

[63] Fabrice Theoleyre, Thomas Watteyne, Giuseppe Bianchi, Gurkan Tuna, Vehbi Cagri Gungor, and Ai-Chun Pang. 2015. Networking and communications for smart cities special issue editorial.Comput. Commun. 58, 0 (2015), 1-3.

[64] Stephen Wang, Yue Wang, Justin P. Coon, and Angela Doufexi. 2012. Energy-efficient spectrum sensing and access for cognitive radio networks. IEEE Trans. Vehic. Technol. 61, 2 (2012), 906-912.

[65] TC Group and others. 2008. SMART 2020: Enabling the low carbon economy in the information age. Global eSustainibility Initiative (GeSI). http://www. gesi.org.

[66] Xinzhou Wu, Sundar Subramanian, Ratul Guha, Robert G. White, Junyi Li, Kevin W. Lu, Anthony Bucceri, and Tao Zhang. 2013. Vehicular communications using DSRC: Challenges, enhancements, and evolution. IEEE f. Select. Areas Commun. 31, 9 (2013), 399-408.

[67] Xin Yuan. 2002. Heuristic algorithms for multiconstrained quality-of-service routing. IEEE/ACM Trans. Netw. 10, 2 (2002), 244-256.

[68] Ruiting Zhou, Zongpeng Li, Chuan Wu, and Zhiyi Huang. 2017. An efficient cloud market mechanism for computing jobs with soft deadlines. IEEE/ACM Trans. Netw. 25, 2 (2017), 793-805.

Received January 2018; revised June 2018; accepted August 2018 